\section{Intersections}

Canadian Journal of Music

Revue canadienne de musique
Intersections CANADIAN JOURAL OF MUSIC
REVUE CANADIENEE DE MUSIOUH

\title{
L'expérience sensible et son devenir abstrait: comment deux amis ont lié d'amitié deux disciplines
}

\section{Nicole Revel}

Volume 30, numéro 1, 2010

URI : https://id.erudit.org/iderudit/1003498ar

DOI : https://doi.org/10.7202/1003498ar

Aller au sommaire du numéro

\section{Éditeur(s)}

Canadian University Music Society / Société de musique des universités canadiennes

ISSN

1911-0146 (imprimé)

1918-512X (numérique)

Découvrir la revue

Citer cet article

Revel, N. (2010). L'expérience sensible et son devenir abstrait: comment deux amis ont lié d'amitié deux disciplines. Intersections, 30(1), 29-64.

https://doi.org/10.7202/1003498ar
Résumé de l'article

Il nous semble important de montrer comment les affinités intellectuelles qui ont uni Roman Jakobson et Claude Lévi-Strauss ont conduit à des liens théoriques étroits entre la linguistique structurale et l'avènement du structuralisme en anthropologie.

Un essai épistémologique mettant en jeu les principales notions opératoires de ces deux disciplines au cours du XX $\mathrm{X}^{\mathrm{e}}$ siècle en Europe et aux États-Unis permettra de comprendre cette émergence des notions fondamentales et des concepts de la pensée et de l'oeuvre lévi-straussienne. Nous proposons de suivre ce développement en quatre temps avant d'accéder aux grands traits de la pensée classificatoire et totémique, à l'analogie comme instrument de perception et de pensée.

Enfin nous témoignerons de deux expériences ethnographiques personnelles en Asie du Sud-est insulaire et de l'influence de nos deux amis dans la pratique d'une recherche en anthropologie linguistique.
All Rights Reserved (C Canadian University Music Society / Société de musique des universités canadiennes, 2011
Ce document est protégé par la loi sur le droit d'auteur. L'utilisation des services d'Érudit (y compris la reproduction) est assujettie à sa politique d'utilisation que vous pouvez consulter en ligne.

https://apropos.erudit.org/fr/usagers/politique-dutilisation/ 


\title{
L'EXPÉRIENCE SENSIBLE ET SON DEVENIR ABSTRAIT: COMMENT DEUX AMIS ONT LIÉ D'AMITIÉ DEUX DISCIPLINES
}

\author{
Nicole Revel
}

Il était une fois deux amis qui ont, par le dialogue, véritablement lié d'amitié deux disciplines, ce qui les conduisit à nous offrir une nouvelle méthode d'analyse des langues naturelles d'une part, des formes de la parenté et des organisations sociales, des classifications du monde phénoménal, des mythes, de la poésie, de la musique et des arts plastiques d'autre part.

J'ai eu le privilège d'être à l'écoute attentive des Paroles données (1984) de l'un, tandis que je lisais avec application Les essais (1963b) et Les leçons de l'autre, au moment précis de l'histoire de la pensée où le structuralisme était à son apogée, entre 1965 et 1970, et dans les années qui ont suivi le retour du terrain que j'avais fait auprès des Montagnards palawan aux Philippines, en 1973.

Je garde de ces années d'études à la Sorbonne et au Musée de l'Homme, puis dans le cadre de l'EPRASS à l'École des Hautes Études en Sciences Sociales (EHESS), au Laboratoire d'anthropologie sociale au Collège de France avec «le cours» et «le séminaire» un souvenir lumineux : alors l'activité intellectuelle intense et partagée se vivait sur le mode du Bonheur. Le livre d'hommage Échanges et communications coordonné par Jean Pouillon et Pierre Maranda (1970) se préparait avec ferveur, l'index des concepts et des noms propres m’avait été confié, travail que j'effectuais dans la bibliothèque, les yeux baissés ... Nous avions le sentiment de vivre une grande aventure de l'esprit. Ce rayon m'accompagne et m'inspire jusqu'à ce jour.

\section{LES PRÉMISSES}

Permettez-moi de reprendre quelques notions fondamentales et de suivre leurs développements.

\section{Le signe linguistique, sa fonction, sa nature}

Le signe évoque un objet immédiat ou un fait d'expérience et se substitue à lui en le re-présentant. Ce representamen (selon la terminologie de Charles Sanders Peirce) permet l'échange entre les hommes, permet la communication. Dès 1867 , je tiens à le rappeler, Peirce, sur la côte Est des États-Unis, avait conçu la théorie du signe, établi le caractère linguistique de la sémantique et fondé la sémiotique. Dès lors plusieurs questions surgissent : les mots sont-ils des images des choses? Qu'est-ce que nommer? 
En vérité nommer, soit représenter par un signe un objet réel, une relation sociale, une pensée religieuse ou politique, une perception, une émotion, un sentiment, un concept, c'est désigner cet objet, mais c'est aussi lier cet objet, cette relation sociale, cette notion représentative greffée sur la vie, à une action et l'insérer dans une vision du monde. Il y a là une véritable appropriation par la langue et la culture considérées. Alors le mot se substitue à la «chose» et l'analyste est en présence de lexèmes, de référents linguistiques distincts des référents dans le monde, une autre interprétation, plus complexe, de la référence. Une mise en correspondance est donc effectuée dès ce moment fondateur du langage. Cet art de mettre en correspondance va avoir des applications à maints niveaux de la pensée et de l'interprétation des pensées d'autrui.

Simultanément, une autre opération s'ébranle : dans le champ partiel de la réalité observée, par exemple l'ensemble des végétaux, l'ensemble des couleurs, dès lors que l'on nomme, on discrimine, j'entends : on distingue ou l'on confond deux catégories monolexémiques ${ }^{1}$.

Si Ferdinand de Saussure a défini la nature du signe linguistique et mis en relief le principe de «l'arbitraire» du signe (Cours de 1911), Roman Jakobson a dépassé cette vue en précisant que le signe "apparaît comme arbitraire» quand précisément on compare les signifiants d'un même signifié dans plusieurs langues. Arbitraire pourrait être traduit par «immotivé», autrement dit, il n'y a pas de nécessité naturelle unissant le signifiant et le signifié. Dans un troisième temps, Émile Benveniste a introduit une nouvelle nuance : «La liaison entre signifié et signifiant doit être reconnue comme nécessaire, ces deux composantes étant consubstantielles l'une à l'autre» (Benveniste 1966, p. 55). En d'autres termes, concept et image acoustique sont inséparables. Jakobson le répètera maintes fois, le signe est le point nodal de la réflexion théorique et ce qui est arbitraire, c'est bien le rapport du signe à la réalité qu'il nomme.

\section{Langue et parole}

La deuxième notion saussurienne qui a influencé les linguistes russes est la distinction entre «langue» et "parole», qui deviendra, avec Roman Jakobson, la distinction entre «code» et "message». "Un processus de communication normal opère avec un encodeur et un décodeur. Le décodeur reçoit un message. Il connaît le code. Le message est nouveau pour lui et, grâce au code, il interprète le message» (Jakobson 1963b, p. 28).

\section{La notion «d'interprétant»}

C'est bien à partir du code que le receveur peut interpréter le message. Mais dans le procès de communication, Peirce a vu plus loin : le signe émis s’adresse à autrui et crée dans son esprit un équivalent ou un autre signe qu'il a appelé «l'interprétant». Et la chaîne des interprétants est indéfinie et infinie. «Le sens d'un signe [émis par A] est un autre signe [créé en B] par lequel il peut être

1 Dans bon nombre de langues de l'Asie, on distingue plusieurs catégories monolexémiques désignant le riz selon les étapes de sa transformation, du riz planté au riz consommable tandis que d'autres langues les confondent en une seule catégorie (Revel 1988). 
traduit». Ou encore : «Tout signe peut être traduit par un autre signe selon lequel il est plus complètement développé». Ainsi, dans le jeu de la communication entre les locuteurs, le délicat processus d'interprétation est clairement posé. Il nous permet aussi de mieux saisir comment la pensée de Claude LéviStrauss par sa propre réception des messages de R. Jakobson, et par la médiation de celui-ci, des messages de Nicolas Troubetzkoy, a pu, à son tour, selon ses propres «interprétants», développer l'analyse structurale des linguistes. En fonction de sa connaissance intuitive et des référents psychologiques, ethnologiques et anthropologiques qui étaient les siens, il a progressivement mis en œuvre de nouvelles notions opératoires dans d'autres champs de l'activité symbolique humaine. Cette projection théorique relève d'une intuition exceptionnelle, d'un sens de l'analogique et d'une interrogation novatrice en psychologie sur les propriétés de l'esprit humain et les processus de la pensée.

Notre compréhension de l'histoire de la pensée en anthropologie et les divers apports théoriques de la linguistique exige un retour en amont.

\section{L'AvANT-GARDE RUSSE ET LES APPORTS DE TROIS CERCLES LINGUISTIQUES EN EUROPE CENTRALE.}

Dans les années 1913-1914 en Russie, le cubo-futurisme et les idées majeures de statique et de dynamique triomphent en peinture et en poésie. Dans ce début du XX $\mathrm{X}^{\mathrm{e}}$ siècle, la créativité dans les arts et la pensée est exceptionnelle et authentiquement révolutionnaire : les sons, la lettre montrent une idée (Klebnikov, Kruchonikh, les frères David et Nicolas Bourliouk, Mayakowsky); la fragmentation, le contraste chromatique, les corrélations de volumes, la facture, soit le travail de la surface avec la matière, sont les nouveaux procédés des peintres cubistes français (Braque, Picasso) et russes (Alexandra Exter, Popova); une sensation pure, le poids, la vitesse, la direction du mouvement dont on ne peut fixer qu'un instant (Kasimir Malevich, El Lissiski), autant de concepts nouveaux qui suscitent une nouvelle esthétique. Toutes nos sensations et les perceptions qui en émanent sont dynamiques. Afin d'être en harmonie avec la vie qui est dynamique, peintres et poètes, compagnons d'esprit, rejettent le passé projeté dans le présent et le futur.

En 1915, Roman Jakobson, encore adolescent, prend l'initiative de créer le MLK, Moskovskij Lingvističeskij Kružok, «le Cercle linguistique de Moscou». Il a dix neuf ans et son objectif déclaré est : «l'étude de la linguistique, de la poétique, de la métrique et du folklore», la première séance du Cercle eut lieu le 2 Mars 1915. Ce cercle rassemble les linguistes et les poètes, dont «le Maître» et l'ami Mayakovsky, mais aussi les philosophes, notamment Gustav Spet, l'élève de Edmund Husserl.

Simultanément à Saint-Pétersbourg, poètes, linguistes et critiques d'art se réunissent en un autre cercle, l'OPOYAZ, Obščestvo po Izučeniju Poètičeskogo Jazyka, la «Société pour l'étude du langage poétique». Victor Shklovsky, Evgenij Polivanov, Ossip Brik, Jakibursky et Fichenbaum vont développer une conception linguistique de la science de la littérature (orale et écrite). À partir de la collection de cent cinquante contes rassemblés par Afanasiev, Vladimir 
Propp procède par induction. Inspiré par les parties constitutives des plantes et de leurs rapports les unes aux autres et à l'ensemble, il soutient : «Il est possible de faire une étude des formes du conte avec autant de précision que la morphologie des formes organiques». Dès 1928, il publie Morphologie $d u$ conte. Le formalisme russe constitue les prémisses de l'analyse structurale que proposera dans les années soixante Claude Lévi-Strauss sur le corpus considérable des mythes amérindiens. Les réflexions de Lévi-Strauss sur cet ouvrage parurent dans le long article «La structure et la forme» sous le premier titre, «L'analyse morphologique des contes russes» en 1960 (repris dans Lévi-Strauss 1973, p. 139-173).

Les liens entre le cubo-futurisme des peintres et le futurisme des poètes se trament dans la conception d'une interrelation dynamique entre différents angles de vue, différents aspects et l'affirmation du pouvoir créateur du langage. En 1915, Jakobson, qui composait alors de la poésie sous le pseudonyme de Aljagrov, fit connaître au poète Klebnikov des incantations et des glossolalies qu'il recueillait dans l'oralité russe. Il percevait dans ces paroles magiques une poésie supra-consciente. En mai 1919, il publia à Moscou une très longue analyse des divers procédés compositionnels de ce grand poète : Modern Russian Poetry: Velimir Klebnikov ${ }^{2}$. Dès 1920, Roman Jakobson encore jeune étudiant quitte la Russie, s'établit à Prague et crée en 1926, avec les Tchèques Mathésius notamment et les Russes exilés, le linguiste Nicolas Troubetzkoy et l'ethnologue Pietr Bogatyrëv, le Cercle linguistique de Prague.

Dès 1925, il avait invité Edmund Husserl à faire une conférence intitulée «Phénoménologie du langage». Alors que Troubetzkoy composait les Principes de phonologie, Grundzüge der Phonologie, la revue CIN (en français, Action) proclame : "Ce n'est pas la genèse, mais la fonction qui est au centre de l'intérêt scientifique actuel». Il s'agit en effet de saisir la cohérence du système et la nature strictement relationnelle et hiérarchique de ses composants. La III ${ }^{e}$ cherche logique (et en particulier le chapitre 2 p. 45-81) : «De la théorie des touts et des parties ", est reprise par les membres du Cercle, comme une méditation de fondement, les fondements du structuralisme que Jakobson définit ainsi : "Chaque ensemble de phénomènes est envisagé comme une unité structurale, comme un système, et la tâche fondamentale est de découvrir ses lois à la fois statiques et dynamiques", ce qui va entraîner les recherches linguistiques en synchronie et en diachronie. Tout comme Charles Sanders Peirce, Edmund Husserl, qui est mathématicien et philosophe, considère que la sémantique fait partie intégrante de la linguistique. Je vois là une autre prémisse aux analyses que mettra en œuvre Claude Lévi-Strauss dans le champ précis de la parenté et de l'organisation sociale, puis dans celui des mythes. Ils avaient eux-mêmes un prédécesseur dans la première moitié du XIX $\mathrm{X}^{\mathrm{e}}$ siècle qu'ils admiraient tous, Wilhelm von Humboldt en qui je vois l'initiateur de la relativité linguistique, généralement attribuée à Boas, Sapir et Whorf. Dans son interrogation sur la relation qui unit le réel observable, le langage et la pensée, Wilhelm

2 Depuis 1973, nous avons de longs extraits traduits en anglais par Edward J. Brown, l'éditeur de Major Soviet Writers. 
von Humboldt a eu l'intuition que le langage représente ce «monde médian à mi-chemin entre les phénomènes empiriques et les structures intériorisées de la conscience». "Notre conception des choses est d'une certaine manière conditionnée par les catégories dans lesquelles elles se situent; en outre les choses placées dans ces catégories, varient de langue à langue». (Humboldt 1974, p. $173^{-178)}$

Cette longue réflexion sur le langage et la pensée, sur la relation étroite qui unit la langue, «la mentalité» et la psychologie et, par voie de conséquence, l'infinie créativité du langage, fut pour le Cercle de Prague une source d'inspiration. Pour les futuristes russes, chaque acte de créativité verbale est une parole. Le parole in libertà de Marinetti va susciter chez les poètes et les linguistes russes, une réflexion différente, beaucoup plus profonde : la poésie du mot est auto-centrée, s'auto-développe, elle est appréhendée comme le langage dans sa fonction esthétique. Dès 1912, le volume publié des poèmes de Klebnikov avait enthousiasmé Jakobson adolescent et cette même année, à Saint-Pétersbourg, ils se connurent. Simultanément, la poésie de Mallarmé «L'Azur», lui parvint à Moscou, alors commencèrent ses premières «interprétations des textes poétiques ». Pour Jakobson, il s'agit bien d'une lecture interprétative, qu'il tentera de rendre rigoureuse en posant les bases d'une nouvelle Poétique. Ses travaux méthodologiques et ses études sur les formes de versification, les tropes ou figures du discours, le rapport entre rythme et syntaxe, les nombreuses analyses de poèmes de tradition orale et des compositions orales-écrites de poètes russes contemporains s'ensuivirent : régularités, parallélismes, symétries, combinaisons d'équivalents sont alors mis en lumière. Plus tard, des poèmes en d'autres langues, (Shakespeare, Dante, etc.) furent analysés. C'est ainsi qu'un jour, grâce à une relation dialogique privilégiée et complice, Roman Jakobson et Claude Lévi-Strauss entreprirent d'analyser de manière ludique avec force dessins «Les chats» de Baudelaire.

\section{Le travail de la métaphore}

Il s'agit de «porter au-delà », de «trans-porter». La métaphore rapproche ce qui est éloigné, ce qui est distant, invisible de prime abord. Elle est donc créatrice, elle est «vive» dira plus tard Paul Ricœur.

Nous sommes confrontés à deux types de métaphores : les métaphores lexicales, qui relèvent de la sémantique du mot, et les métaphores de la phrase, qui relèvent d'une sémantique du discours.

Les métaphores sont potentiellement dans la conscience que les hommes prennent du spectacle du monde. Par un vécu, une expérience, des émotions et les histoires qu'il crée en mettant en relation toutes les entités qui composent le monde, l'esprit humain a une intelligence de celui-ci. La collectivité intériorise et entérine ces paroles, ces récits et ces gestes, car, en eux elle se voit, elle se comprend, elle se re-connaît. Avec la manière inimitable qui est la sienne, Claude Lévi-Strauss a montré la voie aux ethnologues pour comprendre la valence de chaque métaphore, son contenu social, cosmogonique et idéologique, son contenu éthique. 
Il s'agit de travailler non seulement sur le plan de la phrase, du poème, du récit, du mythe, de l'épopée, de tout discours sur le monde, mais encore sur le plan du paysage, de toutes les composantes de la nature (la flore, la faune, les sols, les vents, les marées, le soleil, la lune et tous les astérismes; au niveau des techniques, des objets culturels (bâtons, coquillages, peintures, masques et totems), de l'entrelacs des institutions réelles et imaginaires, des différentes actions rituelles qui mettent en synergie tous les arts de la performance (parole, musique, mouvement, gestuelle, danse).

La geste d'Asdiwal (1958b) m'apparaît aujourd'hui encore comme l'exemple canonique de cette analyse interprétative des mythes, une sorte de préambule à l'océan des variantes et des transformations des «grandes» et des «petites» Mythologiques qui suivront et démontreront la puissance de ses analyses.

\section{La dichotomie métaphore - métonymie}

Dans une théorie du sens, la métaphore - distincte de la comparaison - et la métonymie, apportent un surplus de signification. Ces deux modes d'arrangement sont naturellement dans une relation d'interdépendance qui se manifeste constamment dans l'acte de parole. Roman Jakobson distingue ces deux axes et a montré leur dichotomie. «La fonction poétique projette le principe d'équivalence de l'axe des sélections ou axe paradigmatique, soit le répertoire des termes similaires possibles (synonymes ou antonymes), sur l'axe des combinaisons» (Jakobson 1969, p. 220) ou axe syntagmatique, l'axe de la contiguïté spatio-temporelle (le contenu pour le contenant, la partie pour le tout). Ces deux processus seront repris par Claude Lévi-Strauss et mis en œuvre dans l'analyse de nouvelles formes symboliques, différentes expressions idéelles et artistiques : les mythes, les masques, les rituels, la musique, la peinture, la littérature, etc. Un principe de réversibilité est probablement aussi à l'œuvre et c'est ainsi que des reconversions permanentes peuvent s'effectuer.

\section{Une nouvelle linguistique fonctionnelle et structurale}

Un tournant majeur pour la linguistique s'était opéré en 1939 : l'ouvrage théorique posthume et inachevé de Nicolas Troubetzkoy3, Principes de phonologie, parut. Une nouvelle linguistique, fonctionnelle et structurale, c'est-à-dire « une science sociale qui parvient à formuler des relations nécessaire» (Lévi-Strauss 1958a, p. 40), est désormais proposée. Dès 1942, Jakobson l'enseigna à l'École libre des hautes études de New York. Cette nouvelle méthode d'analyse des faits de langue part de la multiplicité des faits sonores (l'expérience sensible), passe par une opération abstractive et propose désormais d'isoler les sons qui, dans une langue donnée, sont pertinents. C'est là la distinction entre description phonétique et analyse phonologique, entre le niveau de phénomènes conscients et le niveau de leur infrastructure inconsciente - je dirai plutôt, semi-consciente.

3 Le Prince Nicolas Troubetzkoy, décédé en 1938, avait légué son œuvre à l’ami, Roman Jakobson. 
Les notions opératoires sont explicitées et les principaux recours abstraits proposés fondent une méthode d'analyse des sons dans les mots, leur relation et leur valeur intellectuelle (le devenir abstrait). Le mot «phonème» va s'imposer. Troubetzkoy définit la phonologie comme «un structuralisme et un universalisme systématique».

- la notion d'opposition phonologique distinctive et le test de la commutation permettent d'accéder à la notion de phonème. C'est l'avènement du discret.

- Les règles de détermination des phonèmes par le test de commutation effectué à partir de paires minimales sont appliquées. La distinction entre phonèmes et variantes combinatoires (ou allophones) permet de limiter leur définition.

- La classification logique des oppositions distinctives, les corrélations et les faisceaux de corrélations, tout un processus de fragmentation suivi de mises en relation est ensuite opéré.

- Les systèmes phonologiques des oppositions phoniques distinctives se dégagent : les notions de mise en relation et de système sont désormais opérationnelles.

Saussure avait déjà souligné : «Ce qui importe, ce n'est pas l'individualité phonique de chaque phonème vue en elle-même et existant pour elle-même. Ce qui importe c'est leur opposition réciproque au sein d'un système. Tout phonème suppose un réseau d'oppositions avec les autres phonèmes du même système» et formulé la définition canonique : «Les phonèmes sont avant tout des entités oppositives, relatives et négatives " (quatrième leçon, Jakobson 1976, p. 85).

Roman Jakobson assuma le legs de Troubetzkoy et appliqua ce nouvel appareil conceptuel en effectuant l'analyse phonologique des langues slaves en diachronie. Dans sa réflexion sur la vie des langues, on retrouve les lois statiques et dynamiques, la théorie des touts et des parties de Edmund Husserl, les notions de mécanique et de mouvement chères aux peintres cubo-futuristes et aux poètes futuristes, puis à Malevitch. Ainsi il déclare :

La langue en cours d'évolution résout des problèmes internes. Ce serait une conception erronée que de se représenter un système linguistique livré à lui-même, comme condamné à la stagnation ou à l'immutabilité. Les éléments structuraux de la langue s'usent comme les parties de toute structure ou de tout mécanisme. Il faut des réparations qui en renouvellent l'efficacité (Jakobson 1963b).

Il a su travailler à tous les niveaux du langage manifesté en langues «des traits distinctifs en réseaux à l'agencement du texte tout entier» (Lévi-Strauss 1973). Nous y reviendrons. Pendant ses études à la Faculté des Lettres, Roman Jakobson avait rencontré l'ethnologue Pietr Bogatyrëv qu'il a reconnu comme le grand pionnier de l'ethnologie structurale. Plus tard à New York, il a éprouvé une affinité élective à l'égard de ce philosophe qui avait fait plusieurs expéditions dans les immenses paysages du Mato Grosso et de l'Amazonie et observé 
des populations amérindiennes dont, dans les années 1940, on ignorait tout des manières de vivre et des organisations sociales.

Claude Lévi-Strauss avait une intuition structurale parallèle dans le champ de la parenté, soit de la filiation et de l'alliance, de l'organisation des sociétés humaines. Il voit en la linguistique une science qui possède un objet universel, le langage articulé, une méthode homogène quelle que soit la langue où on l'applique, qui repose sur quelques principes fondamentaux dont la validité est unanimement reconnue. Et c'est ainsi que les pensées de nos deux amis qui, à la New School for Social Research, écoutaient leurs enseignements respectifs, se fécondèrent réciproquement. C'est Jakobson qui encouragea Lévi-Strauss à écrire ce qui devint Les structures élémentaires de la parenté (1949). Ce fut le premier ouvrage qui témoigne d'une nouvelle projection de l'analyse structurale dans le champ des sciences sociales. Car Lévi-Strauss perçoit un système de parenté "comme un système arbitraire de représentations", et "parce qu'ils sont des systèmes de symboles ils offrent à l'anthropologue un terrain où ses efforts peuvent presque rejoindre ceux de la linguistique» (Lévi-Strauss 1958a, p. 58). Il me semble qu'on assistait alors à l'avènement d'une intelligence des faits de société et d'une méthode d'analyse, un peu comme en préhistoire on a pu voir en un moment X l'avènement de ce que André Leroi-Gourhan a appelé un «fait technique».

Il va désormais s'agir de découvrir les structures inconscientes de toute organisation humaine et de dégager les lois générales qui y président, et c'est ainsi que Lévi-Strauss a opéré une projection magistrale. Il en vient à assimiler les règles d'organisations sociales à un système de communication : l'échange étant à la base de toutes les institutions humaines, il est tenté par l'interprétation de l'ensemble de la société en fonction d'une théorie générale de la communication. Il distingue trois modes de communication qui ne sont pas à la même échelle et opèrent à des niveaux distincts : la communication des messages (les systèmes linguistiques); la communication des biens et des services (les systèmes économiques); la communication des femmes (les systèmes de parenté) (Lévi-Strauss 1958a, p. 326). Il voit en la prohibition de l'inceste, universellement présente, une forme vide mais indispensable pour que devienne à la fois possible et nécessaire l'articulation des groupes biologiques dans un réseau d'échange d'où résulte leur mise en communication.

Comme le phonème, moyen sans signification propre pour former des significations, la prohibition de l'inceste m'apparut faire charnière entre deux domaines tenus pour séparés. À l'articulation du son et du sens répondait ainsi, sur un autre plan, celle de la nature et de la culture [...] (Lévi-Strauss 1989),

Elles sont consubstantielles l'une à l'autre.

La signification des règles d'alliance, insaisissable quand on les étudie séparément, ne peut surgir qu'en les opposant les unes aux autres, de la même façon que la réalité du phonème ne réside pas dans son individualité phonique, mais dans les rapports oppositifs et négatifs qu'offrent les phonèmes entre eux. (Lévi-Strauss dans Jakobson 1976, p. 12) 


\section{Autre notion opératoire : le binarisme}

Si la fonction d'une langue est de mettre en communication les gens qui la parlent et si le langage est un système de signes, on attend d'eux qu'ils restent distincts les uns des autres. Le binarisme est un outil essentiel pour l'analyse phonologique en linguistique structurale et pour la projection effectuée par Claude Lévi-Strauss en anthropologie culturelle, en particulier l'analyse structurale des mythes.

La substance de ces derniers se trouve dans l'histoire qui est racontée et pour atteindre le sens, il faut le «décoller» du fondement linguistique. Les trois points qui fondent la méthode proposée par Lévi-Strauss sont selon ses termes :

1) Si les mythes ont un sens [...] celui-ci tient à la manière dont les éléments sont combinés.

2) Le langage tel qu'il est utilisé dans les mythes manifeste des propriétés spécifiques.

3) Ces propriétés sont plus complexes que celles qu'on rencontre dans une expression linguistique de type quelconque : elles impliquent la présence des phonèmes, des morphèmes et des sémantèmes. Mais [...] chaque forme diffère de celle qui la précède par un plus haut degré de complexité. $[\ldots]$.

Deux conséquences suivent :

1) Comme tout être linguistique, le mythe est formé de grosses unités constitutives ou mythème. [...].

2) Chaque unité constitutive à la nature d'une relation et toutes les unités constitutives à quelque niveau qu'on les isole consistent en relations (Lévi-Strauss 1973, p. 232-234).

Lévi-Strauss pose alors une nouvelle hypothèse :

Les véritables unités constitutives ne sont pas des relations isolées mais des "paquets de relations» et c'est seulement sous la forme de combinaison de tels paquets que les unités constitutives acquièrent une fonction signifiante. Le système est à deux dimensions à la fois diachronique et synchronique (Lévi-Strauss 1958a, p. 233-234).

Dès 1955, lors d'un symposium sur la notion de mythe (voir Sebeok 1958), à la requête de Thomas Sebeok, Claude Lévi-Strauss a présenté les principes de sa méthode étroitement dérivée de l'analyse structurale en linguistique (1955 et 1973) ${ }^{4}$.

Le principe binaire est également opératoire dans l'organisation des taxinomies. Les opérateurs binaires manifestent en effet une activité de l'esprit, un processus de la pensée repris par la science de l'information. Une fois décrypté, il est relativement aisé de le mettre en jeu dans des analyses aussi bien phonologiques que sémantiques et culturelles.

Il est possible de dégager les phonèmes par le test de commutation et de définir un phonème déterminé, dans une langue particulière, en énumérant ses

4 The Structural Study of Myth, 1955; traduit en français et développé dans La structure des mythes, 1973 . 
traits pertinents. Et ceux-ci sont des traits sonores, articulatoires, dont le jeu oppositionnel, dans le rapprochement de quasi homonymes, va porter les différences de sens. Suivant le vœu de Troubetzkoy, Jakobson a poussé l'analyse au-delà des traits articulatoires, en considérant aussi les traits minimaux des sonorités, les traits acoustiques. L'analyse acoustique reposant sur l'étude des formants entraîne une vision polaire des phénomènes. Jakobson, qui a porté un intérêt constant aux universaux de langage, a alors proposé une théorie linguistique générale, distincte de la description des langues : les traits distinctifs qui forment douze oppositions binaires sont, selon lui, universels, tout comme serait universelle l'opposition entre la marque et son absence. En France, André Martinet a rejeté le binarisme de Roman Jakobson. Par contre, Claude Lévi-Strauss, qui avait des préoccupations analogues et qui se considérait comme le disciple de Jakobson, en a fait une des clés essentielles de sa méthode d'analyse des mythes notamment. Je voudrais simplement faire remarquer que le binarisme et la polarité $+/$ - qui le caractérise, implique toujours un troisième terme intrinsèque au processus intellectuel, au devenir des langues et de la pensée. Ainsi, une opposition binaire peut devenir ternaire (ex : amont/aval/ transverse dans les catégorisations de l'espace en palawan (Revel 1990; 2008) et cette dynamique est soulignée par Lévi-Strauss dans son œuvre.

Dans l'application au corpus mythique, Lévi-Strauss a fait monter à la surface le jeu des oppositions qui sous-tendent ces récits, matérialisant les schèmes abstraits et cachés, superposés et simultanés, qui organisent les séquences (les évènements qui se suivent chronologiquement et qui ne représentent que le contenu apparent du mythe).

La geste d'Asdiwal (1958b, p. 175-234) met en synergie :

plusieurs codes : cosmologique, climatologique, géographique,

topographique

pour faire ressortir une homologie entre des oppositions naturelles :

ciel empyrée / monde chtonien; haut / bas; montagne / mer; hiver / été;

et des oppositions d'ordre sociologiques ou économiques :

filiation / alliance; endogamie / exogamie;

chasse / pêche; abondance / disette.

Que révèle ce jeu d'oppositions?

Entre ces pôles opposés, il n'y a pas de médiateur. Le mythe dans les groupes Tsimshian (4 versions) a la fonction de manifester l'antagonisme des lignées rivales et leur incapacité à le surmonter par une alliance de mariage. Par contre, dans un groupe Kwakiutlt, leurs voisins méridionaux, une société à maison noble, le mythe d'Asdiwal a été emprunté et la version kwakiutl, effectue une synthèse de l'armature du mythe, transforme les valeurs négatives en valeurs positives et fait de l'anti-héros Asdiwal, un héros.

Mais Lévi-Strauss a, en outre, fait des propositions interprétatives par inférence logique, sur des mythes sud-américains que des mythes nord-américains ont explicitement énoncées : RAIE mais aussi PAPILLON FOURMI GELINOTTE ÉCUREUIL, autant d'opérateurs binaires connotant l'intersection de vie et de la mort, qui ont une «ténacité presque incroyable» constate 
Lévi-Strauss, dans la diversité climatique, géologique du vaste espace-temps américain. "C'est l'analyse perceptive, déjà combinatoire et capable d'une activité logique au niveau de la sensibilité, qui, relayée par l'entendement, confère une signification aux phénomènes et les érige en textes. [...] ces textes servent eux-mêmes à articuler d'autres textes» (Lévi-Strauss 1971, p. 501). C'est en cela que «tout système mythique (est) une vaste machine opératoire» et doit être appréhendé comme telle, ce qui a conduit à l'évidence que «les mythes se pensent entre eux ${ }^{5}$ (Lévi-Strauss 1964, p. 20). Dès lors, il s'agit pour Lévi-Strauss de monter les mécanismes de la pensée mythique.

Accordez-moi, en tant que linguiste, de revenir une fois encore en arrière. Que se passait-il dans toutes ces années en France?

Je voudrais évoquer trois linguistes qui, dès 1938, étaient en relation avec le Cercle de Prague et qui ont développé en France l'analyse structurale en synchronie et en diachronie. Il y eut André Martinet d'une part, qui reçut la correspondance de Nicolas Troubetzkoy pour la dérober aux polices allemandes et la conserver. Son analyse sur L'économie des changements phonétiques (1955) est un travail de linguistique fonctionnelle rigoureux. Mais, d'autre part, il y eut, plus proche, Émile Benveniste, qui avec Georges Dumézil et Maurice MerleauPonty, accueillirent Lévi-Strauss au Collège de France.

Je souhaiterais évoquer une personnalité, hors du commun, plus marginale, rationaliste, que Claude Lévi-Strauss a toujours hautement estimée: AndréGeorges Haudricourt ${ }^{6}$.

Haudricourt était en partie autodidacte, très timide, un homme de peu de mots. Dès 1934, jeune ingénieur agronome, il souhaitait se rendre en Asie centrale pour étudier des blés durs. Marcel Mauss l'envoya en Russie pendant un an, à Moscou auprès de Vavilov qui dirigeait le VIR, un institut de génétique sur les plantes (Haudricourt 1987, p. 34-37).

Par goût et par sa formation aux sciences naturelles, cet esprit rationaliste a montré la relation entre la méthode scientifique et la linguistique structurale avec la position fondamentale de la phonologie.

La linguistique structurale n'est autre chose que la forme moderne et consciente d'une discipline ancienne : la linguistique traditionnelle sous son aspect le plus philologique. [...] Cette linguistique moderne met en action le même type de concept abstrait que la physique ou la biologie [...] L'extension des méthodes structurales dans les sciences humaines est possible [...] Mais dans le domaine le plus concret, celui du comportement humain, le retard sur la linguistique est considérable?.

5 Une application de la méthode structurale sur un vaste corpus de mythes et leurs variantes rassemblés auprès des Palawan, a été entreprise et publiée par Charles Macdonald et publiée en 1988 : la comparaison des grands thèmes mythiques a été menée sur des dizaines de variantes recueillies de proche en proche par l'ethnologue sur les thèmes de la morphologie du paysage, de l'éloignement du ciel, de la grande sècheresse, du déluge, etc., et leurs transformations.

6 «C'est un esprit subtil et original qui a su associer dans sa recherche la linguistique, l'histoire des techniques et la botanique. On lui doit des vues très pénétrantes» (Lévi-Strauss 1988, p. 97).

7 Conférence de André-Georges Haudricourt à l'Institut de linguistique, publiée en 1956. 
Haudricourt qui, dans cette citation, montre le retard des autres disciplines en sciences humaines, fut au Collège de France, un auditeur et un lecteur attentif de Lévi-Strauss.

En 1978, il proposa une phonologie diachronique générale, La phonologie panchronique, soit «l'étude de la systématisation des types d'évolution constatées dans l'histoire des langues les plus diverses» (Haudricourt et Hagège 1978, p. 12) ${ }^{8}$. Ainsi fut dégagé un des principes fondamentaux qui guident l'évolution phonique des langues : le principe de conservation des oppositions utiles. Ce même principe qui implique l'idée de transformation se retrouve à l'œuvre entre les variantes des mythes que Lévi-Strauss a tenté de dégager et de comprendre dans le jeu de la pensée humaine.

Je doute que Lévi-Strauss se soit penché sur ces travaux linguistiques hautement techniques d'Haudricourt portant sur une toute autre région du monde, car sa propre entreprise le mobilisait totalement. Peut-être son affinité de pensée avec Jakobson lui suffisait-elle?

Haudricourt formula un très bref commentaire critique sur un résumé de Lévi-Strauss d'un article de Troubetzkoy paru en 1933 dans le Journal de psychologie normale et de pathologie pour lever un malentendu : "Sur le degré d'inconscience des infrastructures " porte sur les quatre temps de la démarche de base de la méthode phonologique. Haudricourt reprend le premier et après une démonstration brillante et très concise conclut :

L'inconscience des infrastructures linguistiques a une valeur statistique, mais le degré d'inconscience varie selon les lieux et les époques et se manifeste à la conscience claire dans certains cas, soit pour des raisons internes (cas des langues flexionnelles où les alternances grammaticales isolent les phonèmes), soit pour des raisons externes (bilinguisme, la confrontation par un même individu de plusieurs systèmes qu'il utilise) (Haudricourt dans Lévi-Strauss et al. 1970, p. 606).

J'évoque avec insistance cette discipline car pour Claude Lévi-Strauss, il s'agissait de transposer la rigueur de l'analyse phonologique structurale à l'analyse des structures de parenté puis à l'analyse des mythes. Sous l'angle de la méthode, il a cherché à systématiser les faits de société, à établir une identité de rapports entre certains faits linguistiques et certains faits culturels.

\section{1952 : LA CONFÉRENCE DE BLOOMINGTON, L'OUVERTURE VERS DE NOUVELLES DISCIPLINES}

Deux textes conclusifs de cette première rencontre interdisciplinaire nous sont restés. Nos deux amis font leurs constats respectifs dans Word9 et dans Supplement to International Journal of American Linguistics (1953), repris dans Essais de linguistique générale (1963). L’un est plutôt circonspect, montre les écueils et

8 Cet ouvrage représente la synthèse de quarante ans de travaux personnels sur les six familles linguistiques de l'Extrême-Orient et en Océanie et les travaux de ses élèves, en étroite collaboration avec Claude Hagège qui apportait la contribution de langues d'Afrique, des Amériques et des langues sémitiques.

9 1953, repris dans Anthropologie structurale, ch. IV, 1958a. 
les fausses pistes qui s'ouvraient alors dans la complexité des rapports entre la linguistique et l'anthropologie, son inquiétude va jusqu'à l'ironie :

Cette conférence m'est apparue comme une sorte de carrousel diabolique où les anthropologues courent après les linguistes, tandis que ceux-ci pourchassent les anthropologues, chaque groupe essayant d'obtenir de l'autre, ce dont celui-ci voudrait précisément se débarrasser. (Lévi-Strauss, dans Jakobson 1963b, p. 8o)

La linguistique structurale était certes le phare théorique, mais une tendance au formalisme se développait, l'anthropologie pour sa part apportait ce sens nécessaire du concret et une sorte de contradiction opposait semble-t-il, les ressortissants des deux disciplines.

Lévi-Strauss propose alors une transposition formelle de certaines notions logiques et opératoires. Il opte pour une position «moyenne» entre la nécessité de séparer les niveaux et la recherche de propriétés communes ainsi que d'éventuelles corrélations entre divers niveaux de réalité, car langue et culture sont deux modalités parallèles mais distinctes d'une activité fondamentale de l'esprit humain, qui fut «le fantôme imprévu» dans ce rassemblement (LéviStrauss dans Jakobson 1963b, p. 81).

En un instant, avec la concision dont il a le secret, Claude Lévi-Strauss donne sa vision de l'avenir des recherches sur la cognition, une interrogation qui sera constante dans sa réflexion sur les faits de langage, de culture et de société, et qui connaît un nouvel essor actuellement.

Roman Jakobson est nettement plus enthousiaste et positif. Il est frappé par «l'étonnante unanimité» qu'il compare à une «structure polyphonique»et, à son tour de prôner un principe fondamental en linguistique: «la nécessité de séparer les niveaux», avec pour objectif suprême : «l'observation du langage dans toute sa complexité» (Jakobson 1963b, p. 27).

Jakobson, quant à lui, se tourne vers deux nouvelles disciplines : la théorie mathématique de la communication et la théorie de l'information et il invite linguistes et anthropologues à une collaboration systématique avec ces disciplines (Jakobson 1963b, p. 28).

Mais il est une distinction importante à faire : linguistes et anthropologues travaillent à des niveaux différents. Ces derniers doivent décrypter «les codes» à travers l'ensemble infiniment complexe, instable et flou des messages et des actions humaines, en contexte.

De ces intuitions parallèles, de ces dialogues entre deux sensibilités et deux esprits si féconds, l'analyse en anthropologie, en sémantique, en poétique, en esthétique, en musicologie s'est enrichie et nous étions parmi les premiers récipiendaires. Il me semble difficile de comprendre les extensions et l'analyse structurale qu'effectua Claude Lévi-Strauss sur des faits de société sans maîtriser l'analyse structurale en linguistique, notamment la théorie générale de Roman Jakobson. Nous sommes en présence de deux pensées théoriques indissociables, je dirai presque consubstantielles l'une à l'autre. L'extension à la sémiologie, à la philosophie, fut un effet de mode et traité avec un silence distant par Lévi-Strauss. 
Si Leonard Bloomfield, qui fut dans un premier temps proche de Troubetzkoy, se détourna de l'analyse structurale, Edward Sapir et les linguistes de Yale avaient commencé à mettre en œuvre ce type d'interrogations, d'enquêtes et de méthode. On assiste alors à un rejet et, simultanément, à un relais pris inattendu.

D’une part, la nouvelle génération de linguistes disciples de Bloomfield à Yale, Charles Hockett et Zellig Harris développent le "distributionnalisme», un formalisme strict avec une attitude anti-mentaliste. Noam Chomsky dès 1957 avec Syntactic Structures, propose une autre recherche structuraliste : le "générativisme", un formalisme plus audacieux encore dans la quête des universaux. Ces nouvelles théories linguistiques n'eurent, je pense, aucune incidence sur la méthode que Lévi-Strauss développait et qui ne pouvait se passer du sens. Dans les années 1963-1965, Roman Jakobson à Harvard fut également fort critique à l'égard d'une linguistique qui se dispensait du sens, tout comme André-Georges Haudricourt à Paris dans les années 1970.

D'autre part, les ethnologues américains - qui sont des hommes de terrain - assimilent et partagent l'apport théorique de la phonologie pragoise et vont appliquer cette méthode d'analyse dans les champs de la lexicographie, de la sémantique, de l'anthropologie culturelle.

Je propose donc que nous nous transportions dans la ville de New York et que nous soyons le témoin de la grande et longue influence de Franz Boas, qui enseigna quarante ans à l'Université Columbia, et de ceux qui furent ses disciples directs Edward Sapir, Alfred L. Kroeber, Herkovitz, Lowie, Ruth Benedict et Margaret Mead pour qui il était un "père», puis Maurice Swadesh, suivi de Marie Hass, de Floyd Lounsbury et de John Lyons.

Ils travaillaient tous sur les langues et les cultures des Amérindiens et essaimèrent vers Berkeley, Chicago et New Haven. Lévi-Strauss les connut presque tous lors de ses deux premiers séjours à New York, puis, en 1952, lors de la conférence de Bloomington et de la conférence qu'organisa Kroeber en 1951: Anthropology Today.

Boas fut l'un des premiers à comprendre la nécessité de la maîtrise de la langue comme moyen essentiel de l'enquête ethnographique. Il décrivit douze grammaires de langues peu ou pas connues et traduisit des milliers de pages de textes en notka, tsimshian et kwakiutl. Il avait intégré parfaitement les acquis de la linguistique pragoise.

Il y a d'une part un nombre limité de sons articulés qui font système, qui s'opposent entre eux de manière distinctive et génèrent un système de phonèmes. [...] Il y a d'autre part, au niveau des mots, un nombre de mots ouvert - mais pas illimité - car les classifications qui unissent les mots sont inéluctables afin que la communication puisse s'établir. [...] Chaque langue a un vocabulaire qui est déterminé par l'univers d'expériences et les catégories appliquées dans ses classifications, chaque langue, par sa grammaire, met en relation ses unités lexicales (Boas 1911).

L'influence pluridisciplinaire de Boas (anthropologie, archéologie, linguistique et textes) rayonnait, linguistes, ethnographes et anthropologues étaient 
tous en communication et développaient la description des langues et des cultures des Amérindiens d'est en ouest, du nord au sud des États-Unis. Chaque année, la Linguistic Society of America fondée en 1925, avait des Summer Schools, le Linguistic Circle of New-York rassemblait linguistes et ethnologues, Word était la revue de ce groupe, tandis que le Summer Institute of Linguistics, (SIL), formait des linguistes missionnaires parmi lesquels Kenneth Pike et Eugene Nida.

Le travail de recueil de textes effectué par Frantz Boas a été une aide infiniment précieuse, indispensable à l'analyse des mythes entreprise par Claude Lévi-Strauss. La geste d'Asdiwal (1958b) et les versions de la Mythologie tsimshian de 1895, 1902, 1912, 1916 puis plus tard, la version Kwakiutl (Lévi-Strauss 1973, p. 175-233 et 1983, p. 220-232), sont indissociables.

Le langage reflète une situation culturelle et suit dans son développement les exigences de la culture. Ainsi les termes désignant des relations : les ethnologues se penchent alors sur les relations de parenté. Les nomenclatures de parenté varient selon le système de consanguinité et d'alliance du groupe social considéré. Par une mise en relation de la nomenclature et des systèmes d'attitudes dans la société globale, au sein de la filiation et de l'alliance, on peut arriver à dégager le système de parenté pertinent.

Boas a insisté le premier sur l'importance de l'activité inconsciente de l'esprit dans la fonction langagière. Il fut suivi par Edward Sapir (Boas 1911; Sapir 1927). Lévi-Strauss reprendra cette trajectoire en proposant de «rechercher les structures parentales inconscientes qu'on peut atteindre à travers des institutions et mieux encore dans le langage $»^{10}$ (Lévi-Strauss 1958a).

Formé à la pensée de Franz Boas, Edward Sapir (1884-1939) contribue à faire de l'Université Yale un centre théorique. Il y avait avant la guerre des linguistes indo-européanistes, sanskritistes, germanistes. Par ses travaux monographiques sur diverses tribus indiennes, Sapir tente d'articuler les relations entre l'inconscient, la personnalité, la langue et la culture : «Il est tout à fait illusoire d'imaginer que l'adaptation des individus au réel peut se faire sans l'usage fondamental du langage ...». «En fait le monde réel est dans une large mesure construit d'après l'habitus linguistique des différents groupes culturels» (Sapir 1951, cité par Hagège 1985, p. 184).

Plus relativiste que Franz Boas, Edward Sapir définit la culture comme «ce que la société fait et pense» et «la langue est une manière particulière de penser ». Reprenant la distinction entre langue et parole, il aspire à élaborer une théorie de la culture, intégrant le comportement individuel et va jusqu'à proposer «une anthropologie de la parole» et «une anthropologie de la gestuelle».

Prolongeant la pensée de Wilhelm von Humboldt (1836-1839) et d'Edward Sapir, Benjamin Lee Whorf (1952) est à l'origine d'une théorie sur les rapports qui existent entre l'univers concret de l'expérience et la structure des langues : la "Théorie de la relativité linguistique» (1940 et 1956). «Le langage est avant tout une classification et une réorganisation opérée sur le flux ininterrompu

10 Conférence à Bloomington, 1952, traduite en français et adaptée de l’original en anglais, «Linguistique et anthropologie», ch. IV, Anthropologie structurale, 1958a. 
de l'expérience sensible ... classification, réorganisation qui résultent en un ordonnancement particulier du monde». Il observe alors que «la segmentation de la nature est un aspect de la grammaire encore peu étudié» (Whorf 1952, p. 240).

Par des mots, plus ou moins distincts, nous assignons un isolement semifictif à des fragments d'expérience. Toutefois, le découpage propre à chaque langue ne concerne pas seulement le lexique mais encore la syntaxe et la morphologie. Ainsi, le système verbal hopi, avec ses neuf voix et ses neuf aspects temporels, conditionne la représentation du temps chez les Hopi. Whorf considère la linguistique essentiellement comme la science des significations. Il meurt prématurément en 1942.

Depuis Boas, ces chercheurs étaient tous membres de l'American Folklore Society. De plus, Paul Fejos, un ami personnel de Lévi-Strauss, dirigeait en 1945-46, le Viking Fund, qui devint la Wennergreen Foundation. Floyd Lounsbury, Claude Lévi-Strauss, Joseph Greenberg et Arthur Kroeber en étaient membres.

\section{LA «NEW ETHNOGRAPHY", PREMIÈre ANTHROPOLOGIE COGNITIVE}

Dans les années 1950-1960, ethnographes et linguistes développaient une nouvelle méthode de travail en se tournant résolument vers l'analyse structurale et les sciences naturelles. Qu'est ce qui est important pour ces chercheurs? L'ethnographie, la linguistique, tout particulièrement, la sémantique et la structure du sens. Floyd Lounsbury et ses élèves, notamment William Sturtevant qui a centré ses recherches sur les sociétés des Amérindiens, les Seneca en particulier, et son ami, Harold C. Conklin, que la Deuxième Guerre mondiale avait détourné des sociétés indiennes et conduit aux Philippines auprès des Hanunóo, s'interrogeaient sur le Sens, sur la nécessité de le cerner rigoureusement afin de mieux traduire les catégories des langues naturelles. Un autre ethnographe, élève de Fred Eggan à Chicago, Robert Bradford Fox, eût un parcours parallèle et resta aux Philippines après la Deuxième Guerre mondiale. Il publia une recherche auprès des peuples negritos, (Fox 1952), sur laquelle Lévi-Strauss fonde son analyse dans le premier chapitre de La pensée sauvage (1962a, p. 3-47).

Fidèles à la tradition de Sapir, ils valorisaient la dimension cognitive du langage. Thomas Sebeok à Bloomington, permit les premières publications en linguistique anthropologique dans International Journal of Linguistic Anthropo$\log y(I J A L)$. Lévi-Strauss était membre du comité de rédaction de cette revue.

En 1949, Fejos enseigna à Yale et parla des Structures élémentaires de la parenté (1949) qui venaient de paraître, Lévi-Strauss s'explique : «Dans un autre ordre de réalité les phénomènes de parenté sont du même type que les phénomènes linguistiques» (1958a, p. 41). La tentative est alors de découvrir les structures inconscientes de toute organisation humaine et de dégager les lois générales qui y président. C'est ainsi qu'il en vient à assimiler les règles d'organisations sociales à un système de communication: l'échange étant à la base de 
toutes les institutions humaines, il est tenté par l'interprétation de l'ensemble de la société en fonction d'une théorie générale de la communication.

Les premiers articles de Conklin dans les années 1950, puis sa thèse en ethnoscience The Relation of Hanunóo Culture to the Plant World (1954), furent très vite connus de Claude Lévi-Strauss par l'intermédiaire de Floyd Lounsbury qui, avec Joseph Greenberg, représentait l'intérêt des chercheurs pour la sémantique et la structure du sens lors des deux conférences majeures : la conférence de New York organisée en 1951 par Arthur Kroeber publiée sous le titre Anthropology Today (1953) et l'année suivante la conférence de Bloomington (1952), organisée par Roman Jakobson.

Par «analyse componentielle» — Zellig Harris créa le mot et explicita cette méthode - il faut entendre une méthode logico-mathématique de description du sens des mots. Le traitement lexicographique des nomenclatures de parenté, envisagées comme des éléments sémantiques en relation mutuelle et formant un champ bien délimité dans l'ensemble structuré du lexique, est systématiquement abordé à l'instar de l'analyse phonologique structurale. Le traitement lexicographique portera un peu plus tard sur d'autres sections du lexique : le vocabulaire des plantes, celui des animaux (poissons, oiseaux, etc.), des couleurs, des maladies notamment. Il s'agit de décrypter les composantes infralexémiques, les traits distinctifs des catégories. Il s'agit aussi de discerner les structures hiérarchiques et les rapports d'inclusion et d'exclusion qui ordonnent les taxinomies en arbres de Porphyre, de comprendre, afin de mieux les traduire, les catégories vernaculaires, les "catégories implicites" 'covert categories', et les modes de penser le concret observable, afin de mieux s'en saisir et d'accéder à un concret pensé, celui des hommes dont on tente de comprendre et de transmettre la langue et les savoirs.

Nous avons des textes canoniques en anglais sur ce traitement lexicographique $(1955,1962,1964)$ et tous les articles de Harold C. Conklin rassemblés et édités par Joel Kuipers \& Ray McDermott parus récemment (2007).

«Dans l'analyse des systèmes de savoirs locaux, il est essentiel de distinguer la réception sensorielle de la perception culturelle. Les variations sont au niveau des cultures plutôt que des différences au niveau des capacités cognitives ${ }^{11}$. Ainsi, les logiques internes comme des systèmes culturels complexes, l'universalité de la créativité humaine sont un objet d'interrogation et d'analyse qui manifestent des affinités de pensée entre Claude Lévi-Strauss et Harold C. Conklin, mais aussi une interrogation partagée par les anthropologues américains de la première «Cognitive Anthropology».

J'ai pris le temps d'évoquer l'École américaine, initiée à Columbia par Boas suivi de son élève Kroeber à Berkeley, mais aussi par Sapir à Yale, où cette «Ethnosémantique» - qui n'est autre qu'une anthropologie-linguistique structurale - a eu un profond développement. Elle situe plus exactement l'univers ethnographique, linguistique et conceptuel dans lequel la pensée de Claude Lévi-Strauss se mouvait aux États-Unis, l'ouverture que ce milieu lui a donné et la réflexion anthropologique que ces magnifiques ethnographies lui

11 Harold C. Conklin, Discours de réception du Prix de la Fondation Fyssen, 1983. 
ont inspiré. Tout cet apport le conduisit à publier le premier recueil d'articles Anthropologie structurale (1958a), suivi de deux ouvrages d'épistémologie et de théorie générale profondément novateurs en 1962, Le totémisme aujourd'hui (1962b) et La pensée sauvage (1962a).

La première rencontre avec Harold C. Conklin date de juin 1961 et eut lieu au Laboratoire d'Anthropologie sociale à Paris. Il fut question de classifications, de noms personnels comme "classes» et de l'article récent alors : «Lexicographical Treatment of Folk Taxonomies», paru en 1962.

\section{EXAMINONS QUELQUES NOTIONS FONDAMENTALES :}

\section{La pensée classificatoire}

Dans le continuum de la nature, il y a des discontinuités naturelles, objectives. Et ce fait est pris en considération par les logiques classificatoires : elles ordonnent un grand nombre d'espèces naturelles qui entourent les hommes dans un environnement donné. Depuis Frazer, un grand débat anthropologique a été centré sur cette question et Lévi-Strauss en a fait la synthèse. Plusieurs niveaux de classifications se présentent et il est possible de passer d'un niveau à un autre de manière rigoureuse. Dans d'autres domaines de la réalité (les couleurs, les saveurs, les maladies) marquées par le continuum qui est inhérent aux phénomènes observés, on est confronté à des catégories floues. Dans la pensée classificatoire, plusieurs systèmes de référence fonctionnent sur des axes différents et peuvent être utilisés simultanément. Une hétérogénéité caractérise ces logiques classificatoires. ${ }^{12}$ C'est là l'indice de la complexité de l'objet et de la densité du réseau des recours linguistiques et abstraits. Dans cette entreprise analytique, «ce qui déroute c'est la nature polyvalente des logiques qui travaillent sur plusieurs axes» [...] «qui font simultanément appel à plusieurs types formels de liaison» (Lévi-Strauss 1962, p. 83).

\section{"Les Totems sont bons à penser"}

Pour un Ojibwa, un «totem» met deux personnes en relation au niveau de la parenté et de l'amitié. C'est le premier sens du mot totem, puis vient la relation avec un animal qui deviendra son esprit gardien (Lévi-Strauss 1962, p. 29 et suiv.). Dans le champ des représentations totémiques, on observe que les nomenclatures des plantes et des animaux permettent à certaines cultures de dénoter des groupes sociaux. Quel rapport y a-t-il entre le système dénommé et le système de dénomination?

En utilisant le nom d'entités naturelles d'animaux, souvent on désigne des gens et des clans, des sections et des sous-sections d'une société clanique

12 En 1830 dans le texte de son intervention au sujet de l'anatomie comparée, Geoffroy St Hilaire avait déclaré contre les fixistes (le baron Cuvier qui fut initialement son ami, avant leur célèbre polémique) : «Les êtres innombrables dans la nature se ressemblent en fonction de certaines relations et se distinguent les unes des autres selon d'autres relations. Toutes les langues et tous les peuples l'attestent», et il ajoute : "La nature ne crée que des individus. C'est nous qui créons les espèces par l'abstraction de différences et la combinaison des ressemblances. À ces combinaisons nous imposons un nom collectif. Les classifications sont très nombreuses et souvent fondées sur des principes opposés.» (St-Hilaire 1830, p. 209). 
exogame. Dès lors, un lien de solidarité et de respect - tabou dans l'adresse, les gestes et les comportements - unit les hommes et les animaux. La Nature et la Culture semblent être dans une parfaite relation d'équivalence.

Pour Claude Lévi-Strauss, les raisons de la dénomination animale doivent s'envisager concrètement. De plus, la discontinuité dans le monde social est différente de la discontinuité dans la nature. Par les noms totémiques, on peut désigner certaines spécificités des groupes dans une société à clans exogames. L'individualité de chaque membre est irréductible, et la rupture entre Nature et Culture est évidente mais la règle d'exogamie entre hommes et femmes dans l'alliance de mariage n'existe pas entre les animaux. Entre les hommes, les lignes des groupes sont parallèles et ne doivent pas se croiser. Néanmoins par l'alliance de mariage, ils se rencontreront nécessairement. Il s'agit donc de réguler cette rencontre et le «totémisme en est le code, le système conceptuel». Il est tout à la fois «pensé» et «agi» (par des prescriptions et des interdits).

Revenons à quelques notions opératoires de Roman Jakobson et à leur reprise par Claude Lévi-Strauss.

\section{L'analogie comme instrument de perception et de pensée, formule canonique}

Une identité de rapport (A est à $\mathrm{B}$ ce que $\mathrm{C}$ est à $\mathrm{D}$ ) fonde les notions d'homologie, de ressemblance, et d'équivalence. Pour cela il faut, entre les entités, des traits communs et des différences. Aussi les entités sont-elles différentes, mais sous un certain angle de vue, on peut les considérer comme équivalentes, LéviStrauss parle plutôt «d'équivalence partielle».

Les objets physiques et les phénomènes peuvent interagir. Les hommes les observent et les manipulent en formulant des représentations qui manifestent cette relation d'équivalence partielle. Le raisonnement par analogie est donc un rapport de ressemblance établi par l'intelligence ou l'imagination, une relation de similarité, un processus de pensée universel très productif. Avant d'accéder à une métaphore, l'analyse dégage une similitude réelle de rapports, de fonctions et de visées. Ce raisonnement est à la fois poétique et scientifique : poétique, car les métaphores et les comparaisons sont sa production; scientifique, car le langage de la description et/ou l'interprétation théorique des sciences la mettent en œuvre également. Les philosophes du langage considèrent que le modèle est au discours scientifique ce que la métaphore est au discours poétique et spontané. (Black 1968, p. 23; Hesse 1965, p. 302-06)

Les ethnographes nous donnent les exemples illustrant ces modes de percevoir et de concevoir le monde. Claude Lévi-Strauss a démontré leur puissance opératoire par le biais de l'analyse structurale de faits concrets dans diverses sociétés humaines, en essayant de capter les processus de la pensée et les logiques qui ordonnent les plantes et les animaux ainsi que certains faits de société. En anthropologie, le travail analytique consiste à déceler les relations analogiques privilégiées par différentes cultures et leurs doubles torsions (Lévi-Strauss 1973, chapitre VIII), afin de comprendre le système symbolique qu'elles ont créé. Grâce à ces systèmes, les hommes agissent et conçoivent le monde qui les entoure. Ils vivent en son sein dans une relation intime selon une éthique 
et une esthétique tant il est vrai que : «Le sens ne se décrète pas, il est partout». «Signifier n'est jamais qu'établir une relation entre les termes» (Lévi-Strauss, 1962, p. 590).

\section{LA LINGUISTIQUE ANTHROPOLOGIQUE COMME DISCIPLINE À PART ENTIÈRE}

J'ai intériorisé ces enseignements, ces notions opératoires, ces outils conceptuels et je les ai partiellement mis en œuvre dans mon travail monographique sur une langue et une culture orale austronésienne, dans le Monde insulindien. Ainsi j'en suis venue à développer, à mon tour, une linguistique anthropologique ou une ethno-linguistique, une ligne de force qui émanait de la pensée de nos deux amis à New York. Elle était déjà mise en œuvre par les travaux des ethnographes linguistes de la New Ethnography à Yale, rassemblés par Dell Hymes en un ouvrage fondamental publié en 1964, Language in Culture and Society, puis One Hundred years of Anthropologial Linguistics de Floyd G. Lounsbury en 1968, à Harvard, suivi de Cognitive Anthropology : Readings édité par Stephen Tyler en 1969 et la magistrale compilation : Folk Classification. A Topically Arranged Bibliography of Contermporary and Background References Through 1971, publiée par Harold C. Conklin à Yale en 1980.

Je voudrais aborder maintenant mes propres travaux, et tout d'abord l'inspiration liée à "La science du concret», tel que l'ouvrage La pensée sauvage nous l’a révélé.

Auprès des Montagnards palawan dès 1970, les recherches sur la connaissance de la nature furent entreprises tout naturellement, si je puis dire, elles s'imposaient à moi par les actions et les préoccupations quotidiennes des hommes, mais aussi par l'influence qu'a exercé sur moi la «pensée sauvage» dans le sens étymologique de "au plus près de la nature».

Une formation théorique intense avant le départ, comprenant l'initiation à l'analyse phonologique sur les langues des cinq familles linguistiques de l'Asie et l'enseignement de la relation avec les sciences naturelles que nous prodiguait André-Georges Haudricourt - auquel Lucien Bernot et Georges Condominas m'avaient confiée - et Jacques Barrau, fondateur de l'ethnobiologie au $\mathrm{Mu}$ séum d'Histoire naturelle. Tous deux avaient séjourné et travaillé avec Conklin à Yale, et m'invitaient à suivre cette voie. Haudricourt, comme Lévi-Strauss, était un fervent admirateur de Jean-Jacques Rousseau. Il manifesta son admiration par la réédition en 1983, des Huit lettres élémentaires sur la botanique écrites de 1771 à 1773 et adressées à Madame Delessert, édition augmentée de Fragments pour un dictionnaire des termes d'usage en botanique (p. 97-149). Haudricourt assistait fidèlement au cours sur les Mythologiques les yeux fermés, l'oreille en coin, car une estime intellectuelle réciproque unissait ces deux penseurs rationalistes et structuralistes, en silence ... Haudricourt était trop timide et, de son propre aveu, ne se sentait "pas assez philosophe» pour dialoguer avec Lévi-Strauss.

Pour ma part, j’avais intégré deux préceptes de Claude Lévi-Strauss : 
Il faut viser au cœur de la culture et pour chaque culture, chercher à atteindre ce qui lui appartient en propre : mythes, rites, langage, c'est-à-dire ces domaines où les oppositions sont à la fois isolables et inconscientes (1984 p. 33).

et

Seule l'expérience peut permettre de découvrir les principes qui guident les classifications indigènes.

\section{L'EXPÉRIENCE INTIME VISUELLE, L'EXPÉRIENCE INTIME AUDITIVE}

Nuages de mer, îles de ciel, terres aux multiples contours flottant à fleur d'eau, Palawan et les archipels de Sulu invitent à la contemplation. Montagnes et forêts riches de milliers d'espèces, - nous sommes dans un «berceau cultural» selon la qualification de Carl Sauer ${ }^{13}$ — abris fragiles dans le cosmos, Nägsalad, «le Tisserand», par sa seule pensée réfléchie, a créé un bel univers à deux fois sept niveaux superposés.

Découvrant au jour le jour le savoir encyclopédique des Montagnards sur le monde phénoménal, j'ai entrepris un travail de longue patience, qui n’avait d'égal que mon plaisir à collectionner les plantes, les coquillages, les insectes, les oiseaux, les poissons, les objets et les histoires, et j'ai été entraînée dans une aventure insoupçonnée.

Il s'agissait précisément de comprendre, afin de la traduire, la «science du concret» des Palawan, il s'agissait pour eux d'identifier, de nommer, de classer et pour moi de décomposer, de restituer et de traduire, un savoir empirique partiel très dense, logique et abstrait, cosmogonique et global.

Pour mener à bien cette entreprise ethnographique, lexicographique, taxinomique et sémantique, j'ai mis en œuvre diverses techniques ostensives. De plus, j'étais à l'écoute de toutes les formes d'interlocution. Simultanément, dans la dynamique du vécu au fil des jours, j'effectuais une analyse structurale de la langue palawan, une langue orale de la famille austronésienne (Thèse de III ${ }^{\mathrm{e}}$ cycle en 1974, publiée en 1978), et cela me conduisait nécessairement vers une ethnographie de la parole.

Une translation entre les objets naturels et les signes qui les représentent s'opère. Alors s'instaure entre le signe linguistique et son référent dans le monde, un rapport paradoxal de solidarité et de liberté relative, puisqu'elle n'est que la manifestation contingente des valeurs, des modes, des conventions de la culture considérée, à un moment donné.

Le regard analytique se déplace vers des niveaux de complexité croissante du sens et des représentations symboliques, qui conduisent à cerner une conception métaphysique du cycle de vie et de mort, une biocénose. Les divers champs lexicaux, les pratiques, les actions de la vie quotidienne sont également captés, décrits, transcrits, traduits. Le contexte et les circonstances appellent à

13 Voir à ce sujet Jacques Barrau 1974, p. 17-39. 
des applications et des manipulations incessantes des représentations constitutives de la culture, qui se déploient dans divers champs sémantiques, toujours partiels et dans les taxinomies, ces ordonnancements hiérarchisés d'objets nommés finis.

C'est cette interrogation plus idéelle de représentations collectives, que l'ethnographe - ethnologue - linguiste a pour tâche de cerner, d'analyser, et j'ajouterai de traduire, ainsi que Lévi-Strauss nous l'a toujours montré par ses analyses lors de son enseignement.

Harold Conklin, en août 1970, eut la générosité de nous emmener, Charles Macdonald et moi-même, en pays hanunóo; plus tard, il nous accueillit en pays ifugao. Sa profonde estime pour la personne et la pensée de Lévi-Strauss, alliant le sens du concret, la perfection du détail, l'impératif de l'expérience in situ, doublée de la maitrise des langues et de l'analyse structurale en lexicographie, assurait un continuum théorique. C'est ainsi que la pratique de "l'ethno-linguistique » s'est consolidée dans mes travaux et que j'ai réalisé, dans la fluence de la vie et d'un long travail, une histoire naturelle, Jacques Barrau me l'apprit un jour.

Accordez-moi l'évocation d'une autre expérience sensible et son devenir abstrait. Dès l'arrivée dans le piémont, le paysage sonore est autre. La petite musique des choses vous enveloppe : chants des oiseaux, stridulations des insectes, souffles des vents, rumeurs du torrent, cris enjouées, autant de mélodies, de paroles et de chants que ces phénomènes inspirent aux enfants, aux adultes.

L'accueil au visiteur, épuisé par la marche, est des plus courtois. On lui donne à entendre le chant d'une épopée, la nuit durant. Quand le soleil étendra ses premiers rayons, l'interprète devra s'arrêter et l'intrigue se dénouer.

Ce n'est pas la biographie du héros qui est fondamentale, c'est plutôt son action, la qualité qui identifie sa parole, sa personne, sa présence au monde, son art d'évoluer en société ou ses manquements que l'intrigue va se charger de corriger. L'épopée chante une crise du monde, de tous les mondes possibles, et sa résolution, le plus souvent grâce à une alliance de mariage.

Pendant la dernière décennie du $\mathrm{XX}^{\mathrm{e}}$ siècle, lors d'un programme de recueil et de sauvegarde dont j'ai eu l'initiative, nous avons constitué une archive multimédia sur quatre types de supports (audio, audio-vidéo, photos, et mémoire informatique des chants en langues vernaculaires et traduits en une langue véhiculaire (anglais, français ou tagal). Quatre-vingt-cinq épopées en quinze langues différentes plus une à Sumatra sur les cent douze manuscrits établis ont été recueillies en collaboration avec vingt chercheurs et/ou membres des communautés indigènes. L'ensemble constitue une base solide pour un comparatisme futur et est une archive multimédia consultable sur la toile depuis la conférence «Songs of Memory » ${ }^{14}$.

L'analyse de quatre récits ifugao au nord de Luzon chantés par une narratrice accompagnée par un chœur de femmes, hudhud, et de sept récits palawan tultul, chantés par un homme a capella, enregistrés, transcrits et traduits,

14 Du 20 au 22 Janvier 2011 : <http://epics.ateneo.edu/epics>. 
révèle que malgré un système de parenté de type cognatique (ou indifférencié) et une organisation sociale différente ${ }^{15}$, l'alliance de mariage idéale est régie par un système d'échange symétrique, paritaire, à savoir, deux hommes échangent leurs sœurs et deviennent ainsi beaux-frères. Il s'agit là d'un échange symétrique qui n'atteint pas encore la forme réitérée de «l'échange restreint», soit le troisième mode de communication par échange de femmes dans la théorie de l'alliance des Structures élémentaires de la parenté (1949), car il n'implique que deux partenaires, puis s'arrête.

Dans ce travail d'analyse des représentations idéelles de l'épopée, l'analyse de La geste d'Asdiwal (1958b) m'a profondément inspirée (voir Revel 2000, 1983 et Revel et al. 2005).

En observant les topoi et en analysant la deixis d'une épopée de chacune de ces cultures, j'ai eu aussi l'idée de faire un petit «bricolage».

Le récit est conçu comme un espace linéaire avec des lieux où les personnages évoluent. Les hameaux, mais aussi la forêt, la mer, l'espace médian, sont autant d'espaces géographiques et symboliques mais aussi de bornes, où les actions, les voyages et les épreuves des actants, ont lieu, soit les points nodaux de l'action séquentielle. L'espace ainsi balisé imprime une forme structurante au récit. Paul Veyne (1996, chapitres III et VI), puis Paul Ricœur avec Mimèsis II de Temps et récit notamment, ont analysé la notion d'intrigue et de mise en intrigue.

À cet axe horizontal, qui est celui des séquences du récit, Claude Lévi-Strauss aurait ajouté l'axe vertical des schèmes, superposés et simultanés qui organisent ces séquences en un jeu plus abstrait d'oppositions, et celles-ci fondent la rationalité de la pensée. Le jeu des paradigmes vient de surcroît.

Quant à moi, confrontée à ces longs récits chantés que j'établis, je m’interroge sur une autre dimension cognitive: identifier les repères de la mémoire narrative des poètes-narrateurs lors de la performance. J'observe que les interprètes naviguent et guident leurs auditeurs dans un espace-temps imaginé précis. Intrigue et deixis spatio-temporelle révèlent le jeu relationnel qui peu à peu se précise entre un «texte mental ${ }^{16}$ et une «carte mentale».

La mise en intrigue, cette séquence d'actions reliées par une relation de causalité ou d'implication, est indissociable de l'action. Elle exprime, dans son évocation, le mouvement. La multiplicité des voyages des héros et de leurs adjuvants sur terre, en mer, dans l'espace médian, l'atteste. De voyage en voyage, un parcours initiatique se déploie et conduit les principaux actants vers une résolution de la crise et l'avènement d'un monde nouveau harmonieux, reconstruit. Pour le poète-narrateur, il s'agit bien de nouer et de dénouer une

15 Il s'agit d'une société divisée en trois classes chez les Ifugao au Nord de Luzon d'une part (Conklin 1980) et, d'autre part, d'une société acéphale et égalitaire au Sud de Palawan (Macdonald 1977) ainsi que chez les nomades marins Sama Dilaut de Tawi-Tawi (Arlo Nimmo 1972 et 2001), (Martenot 2005).

16 La notion de " texte mental » a fait l'objet du premier atelier de réflexion qui s'est tenu à Turku en 1993, et rassembla les chercheurs dans le cadre d'un séminaire international sur l'épopée que je coordonnais dans un des Programmes de la Décennie pour le Développement Culturel (1990-2000) à l'Unesco : Étude intégrale des routes de la soie: Routes de dialogue. Les conférences furent rassemblées et publiées en 1996 par Laurie Honko dans un numéro spécial de Oral Traditions. 
intrigue qui se trame entre des actants, leurs adjuvants et leurs opposants. Il est possible de représenter une histoire comme un réseau de relations causales, soit un lien entre deux évènements, tant du côté de l'interprète que de ses auditeurs. Une épopée est faite «d'une succession pertinente, une série d'incidents et un aspect temporel caractérisé par une intégration, une apogée et une fin, de telle sorte que l'histoire reçoit une configuration très particulière. Si bien que composer une histoire est, sous l'angle de la temporalité, dessiner une configuration à partir d'une succession» (Ricœur 1977). Les trois opérations successives : préfiguration - configuration - re-figuration, soulignent les liens du récit et de l'action avec le temps. C'est là le cercle de la Mimèsis.

Dans la tentative de capter la vision intérieure du barde, je propose donc de dessiner un graphe orienté : il représente les actions successives des divers protagonistes nouant et dénouant une intrigue dans l'espace-temps. Alors, pensée active, attention et mémoire se conjuguent pour intérioriser l'intrigue dans l'instant éphémère de la proferatio et la chaîne de la transmission s'ébranle. Au-delà du continuum linéaire de l'histoire, il y a une pensée topologique. Les Montagnards Palawan parlent des "chemins", dalan, et je pense qu'ils sont d'une certaine manière conscients du noyau central qu'est l'armature et des connections causales avec de multiples voies de rappel, car des liens nombreux unissent les composantes d'une histoire. Ainsi, par les «chemins» de l'action racontée, l'aède transmet-il un modèle de parcours.

\section{La «composition mémorative»}

Dans une situation caractérisée par l'absence de tout support écrit, dans le cadre d'une "oralité pure» dirait Paul Zumthor, re-présenter l'intrigue par un graphe, n'est pas autre chose que de re-présenter un réseau de relations causales afin de favoriser l'intégration d'une intrigue dans l'espace temps de

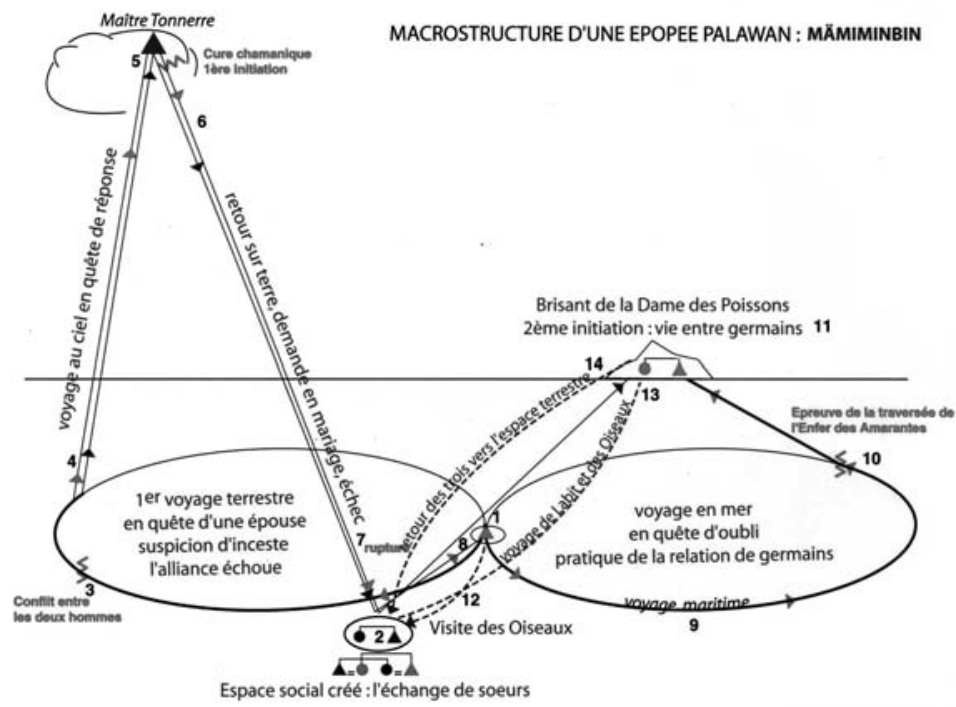


la performance et sa mémorisation. Cette activité se rattache à une activité de traitement de texte (mental). Dans une chaîne de transmission orale-aurale, cette «carte» cognitive, si l'on m'accorde ce calque, s'inscrit dans la mémoire des auditeurs. Mais je propose en outre de considérer que ce récit est tout entier dans une praxis de l'espace-temps du barde et de ses auditeurs, un espace et un temps vécu par chacun d'eux.

Il s'agit en effet pour les auditeurs de décomposer et de re-synthétiser. Ces longes histoires sont aisément intériorisées, puisqu'elles configurent le vécu des Montagnards palawan, ifugao ou des Sama Dilaut, les nomades de la mer, dans l'espace géographique, techno-économique, sociologique, cosmogonique et moral qui les entoure, respectivement.

Voici donc deux graphes synoptiques, une vue à la fois analytique et synthétique, de l'armature et des configurations symétriques qui organisent chacun des deux récits en un tout cohérent et qui chantent précisément un échange symétrique. C'est là, je pense, la carte mentale, implicite à la pensée du barde, qui soutient de son armature la mémoire de ce sage-poète-musicien, qui soustend au niveau séquentiel une composition qui est re-création lors de chaque performance et permet aux auditeurs de re-synthétiser le récit et de peut-être, un jour de devenir aède, tant il est vrai que «Les valeurs ne se réduisent pas à ce que les hommes croient et disent; elles tiennent aux instruments dont les hommes se servent pour penser» (Lévi-Strauss 1959-196o, p. 34).

Ma dette est bien grande à l'égard de chacun et, en particulier, de nos deux amis. J'ai esquissé pour vous l'horizon de pensée de Claude Lévi-Strauss. Pour nous ethnographes, ethnologues, anthropologues, la pertinence scientifique de son œuvre est telle qu'il sera toujours nécessaire d'y revenir, elle est, outre sa beauté rationnelle et littéraire, un inépuisable réservoir pour la pensée anthropologique.

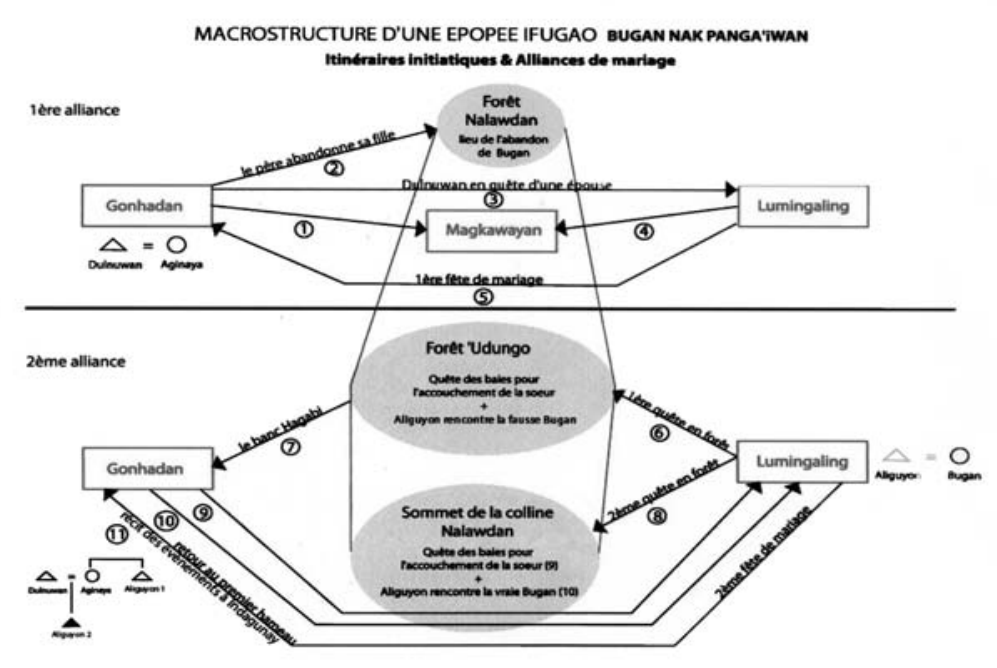


Pour conclure, je vous propose un nouveau petit bricolage. Prenons l'image de la quatrième de couverture de L'Homme nu (bronze d'Amlash) et faisons une lecture interprétative.

Un homme monte au ciel, serait-ce Asdiwal, poursuivant l'Ourse qui l'entraîne vers la demeure de Soleil, son père, et va devenir Étoile du soir, sa première épouse?

Il a franchi la couche des nuages et atteint le dernier échelon d'une échelle fragile. Il regarde et «le ciel lui apparait comme une vaste prairie verte et fleurie», à l'image de la terre dont il aura un jour la nostalgie, nous dit le mythe.

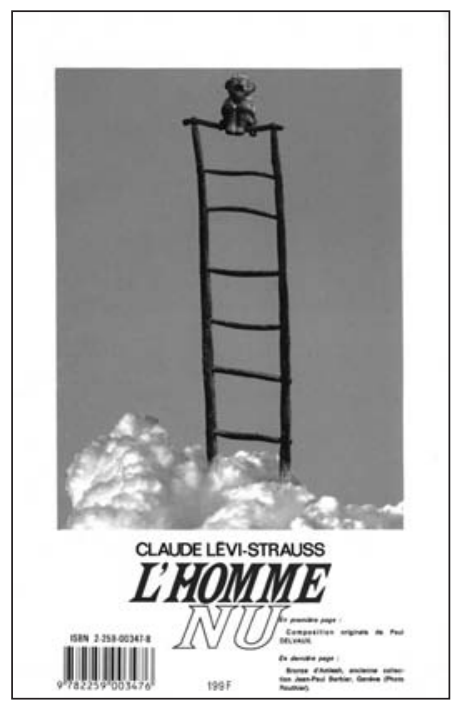

Grâce à sa chasse - poursuite audacieuse et à son ultra-voyance, il a semblet-il accompli un Voyage chamanique. Dans l'empyrée, il nous regarde, distant, calmement assis, tenant ses jambes pliées entre ses bras pour garder l'équilibre sur cet ultime échelon. Je perçois là une signature mettant un terme à cet immense effort de savoir, de mémoire, d'analyse et de synthèse interprétative, d'écriture et aussi d'enseignement qui dura neuf ans (1961-62 à 1970-71), la «tétralogie des grandes mythologiques».

Continuons ce bricolage et ajoutons un 'step ladder poem'. J'ai choisi : «The Most Extraordinary Adventure that Happened to me at the Rumyntsev Dacha, Pushkino, Akulova Gora, on the Yaroslav Railway Line ... SUN» de Vladimir Mayakovsky, dans le livre façonné par El Lissitzki For the Voice. En l'absence de la voix magnifique de Mayakowsky, je vous donne à lire en russe la quatrième page de ce dialogue entre Soleil et Poète et la belle traduction anglaise de Peter France de l'Université d'Édimbourg présentée en regard. 


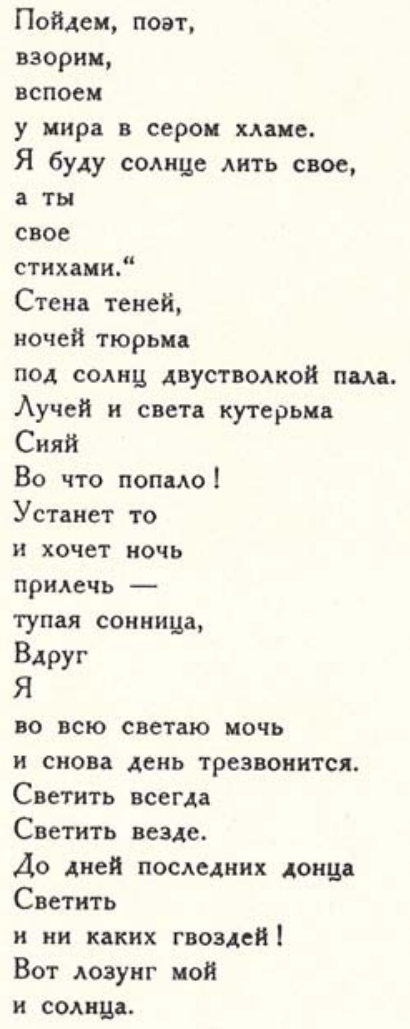

Come, poet

let us shine

and praise

in this grey dump of ours.

I shall keep pouring out my rays, and you

can shine

in verse."

The wall of dark, the prison of nights,

our double barrel can conquer.

Let the dance of rays and light

shine out

with maximum power!

If he's sleepy

and feels like a night

of rest,

a little lie-down,

then

I

will shine with all my might and day will ring over the town. Shine everywhere.

Shine all the time.

Till the last minute comes.

Shine

with a vengeance! Shine and shine! That's my motto,

and that's the sun's.

\section{Remerciements}

Le Dr Harold C. Conklin a eu la générosité de m’accueillir à Yale University, la veille de cette conférence en l'honneur des 100 ans de Claude Lévi-Strauss. Il a partagé avec moi ses souvenirs et m’a prodigué encore une fois son grand savoir théorique, qu'il soit ici profondément remercié. 


\section{Annexe 1 Mämiminbin :}

Liste des oppositions entre les actants, les adjuvants et les milieux où l'action se déroule.

\begin{tabular}{||l|l|l||}
\hline \multicolumn{1}{|c|}{ Espaces géographiques } & \multicolumn{1}{|c|}{ Environnements } & Flore, faune, phénomènes \\
\hline $\begin{array}{l}\text { Hautes-Terres } \\
\text { Mer des Nattes } \\
\text { Atmosphère }\end{array}$ & Forêt et rivière & Oiseaux, sangliers \\
& Brisants sur mer d'huile & Poissons, Tandayag, \\
\hline
\end{tabular}

Il y a sept personnages, mais six cellules mélodiques distinctes seulement. Tandayag n'a pas de parole. Il est un adjuvant abyssal silencieux dès lors qu'aucune faute entre consanguins n'est commise. Il est efficace et ramène les héros vers le littoral, sur la terre car le projet d'alliance, il le voit bien, est beau, sage, égal, paritaire, harmonieux en tous points.

\section{Les héros}

Mämiminbin Jeune homme des Hauts, d'une grande vitalité, d'une grande beauté physique, emporté, agressif et sans manières.

Läbit

Jeune homme des Hauts, d'une grande beauté physique, pondéré, honnête, pacifique et courtois.

\section{Les dames}

Ariq ni Läbit Jeune fille des Hauts, d'une grande beauté physique, colérique et obstinée, sans maturité et sans maîtrise de soi, refusant tout ou exigeant tout. Personnalité capricieuse.

Ämpuq ät Säraq Dame des Poissons d'une grande beauté physique et psychologique.

Sa demeure est dans l'espace maritime.

Sereine, grande maîtrise de soi et des situations, prenant des initiatives, bonne médiatrice. Personnalité conciliante.

\section{Les adjuvants}

Balud-Putyän

Pigeons impériaux, Pigeons-Pagodes.

Ils appartiennent au monde aérien mais sont aussi des oiseaux domestiques sur la terre et des conseillers.

Kuyäw

Grand Père Tonnerre, Divinité de l'espace médian, doté d'ultra-voyance. Gardien du monde.

Tandayag Dragon, divinité chthonienne et abyssale, silencieux, mais doté d'ultra-voyance. Gardien du monde. 


\section{Voyages des personnages}

La notion d'espace est indissociable de l'action des héros et celle-ci est étroitement liée au voyage. Ce n'est pas la biographie du héros qui est fondamentale, c'est plutôt son action, son mode d'agir et de se comporter, la qualité qui l'identifie et son évolution au cours de l'intrigue.

La trajectoire de Mämiminbin dans l'espace clos de l'épopée n'est autre qu'un passage initiatique : des mauvaises manières, par une série d'épreuves, il accède aux bonnes manières, de la malséance à la bienséance, selon la morale palawan, le code qui l'exprime et régit la société.

Dans la nature et la surnature, sept personnages évoluent : deux hommes, Mämiminbin et Läbit, une Puissante, Ämpuq ät Säraq, la «Dame des Poissons», et leurs adjuvants sylvestres et maritimes, les Pigeons-Pagodes et le Dragon, Grand-Père Tonnerre, veillant dans l'espace médian. La petite sœur de Läbit ne quitte pas la maison qui deviendra le hameau, le centre d'un espace social construit. Les épreuves sont symbolisées par un éclair.

Pour arriver à construire un espace social harmonieux, quatorze voyages - deux fois sept voyages - ont été nécessaires, ainsi que l'atteste la liste ci-après.

1) Quête en épouse de Mämiminbin dans les Hautes-Terres. Rencontre de la petite sœur de Läbit.

2) Descente précipitée vers la rivière du frère aîné volant à son secours, Läbit.

3) Chasse-poursuite autour du monde entre Mämiminbin et Läbit.

4) Voyage chamanique de deux hommes vers l'espace médian, demeure de Grand-père Tonnerre.

5) Retour de Läbit sur la terre, accueilli par sa sœur cadette.

6) Suivi du retour de Mämiminbin et visite à la maison de Läbit pour réitérer sa demande en mariage.

7) Retour de Mämiminbin à sa maison esseulée, après avoir été éconduit.

1) Nouveau départ de Mämiminbin en quête d'oubli et prospection. Épreuve franchie de l'Enfer des Amaranthes, un espace incandescent : un monde nouveau s'ouvre, l'espace maritime.

2) Voyage des Pigeons-Pagodes en quête d'un nouveau maître. Arrivée à la maison de Läbit.

3) Voyage aérien de Läbit et des Pigeons-Pagodes vers le monde maritime, en quête de Mämiminbin.

Épreuve franchie de l'Enfer des Amaranthes. Arrivée à la demeure de la Dame des Poissons.

4) Vol de retour incognito des Pigeons-Pagodes vers la maison de Läbit.

5) Voyage de retour vers le littoral des trois personnages, Läbit, la Dame des Poissons, Mämiminbin grâce à Tandayag, le Dragon : retour à la maison 
de Läbit et de sa sœur cadette, la seule à ne pas voyager conformément à l'Adat.

6) Retour sous-marin du Dragon sans sa maîtresse, la Dame des Poissons.

7) La Dame des Poissons intègre la vie sur la terre, l'alliance est scellée entre les quatre personnages en tous points harmonieux. Un espace social se forme ainsi dans un monde paisible et empreint de courtoisie en 15.

\section{Annexe 2 Bugan fille de Panga'iwan :}

Ce hudhud est construit en deux épisodes majeurs qui reflètent une symétrie nécessaire dans l'alliance de mariage idéale, ici encore l'échange de deux sœurs entre deux hommes qui deviennent beaux-frères dans le groupe des Kadangyan, «les Riches». La parité est fondamentale dans la société ifugao, organisée en trois strates. Ici l'exigence dans l'alliance porte sur la strate sociale la plus élevée, un groupe très exclusif. Cette parité est manifeste par les parures, les attributs, les déplacements et les rituels qui unissent peu à peu les personnages.

Deux hommes vont échanger leurs sœurs respectives et cette alliance sera harmonieuse et porteuse de paix entre deux villages Gonhadan et Lumingaling.

L'intrigue se noue donc entre trois villages, espace social (encadré), et trois lieux dans la forêt, espace initiatique (encerclé).

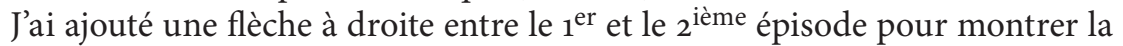
nécessité des deux alliances et la succession des rituels à accomplir pour que l'échange paritaire soit fondé et s'accomplisse et que le cercle se ferme, que l'intrigue se dénoue harmonieusement au village du point de départ.

Une petite fille est rejetée par sa mère alors que cette dernière attend un deuxième enfant. Chez les Ifugao, il est important d'avoir un garçon, puis une fille. Aussi demande-t-elle à son époux de prendre leur fille et de l'abandonner en forêt. Il hésite, mais accomplit cet acte. Il dépose l'enfant sur un arbre narra sans omettre toutefois de la parer d'un beau collier, emblème de son statut social, qui permettra, plus tard, de l'identifier.

Alors qu'elle pleure de frayeur sur l'arbre agité par le vent, elle est sauvée par un homme qui deviendra son père adoptif et qui l'élèvera.

Toute l'intrigue porte sur une perte d'identité et son recouvrement, met l'accent sur l'échange symétrique entre les partenaires, mais également - dès lors que l'on appartient au groupe des Kadangyan-, la nécessité d'accomplir les rites successifs qui précédent l'alliance paritaire et permettent l'avènement d'une bonne filiation. Ces traits essentiels figurent à gauche.

\section{RÉFÉRENCES}

Arlo Nimmo, Harry. 1972. The Sea People of Sulu. A study of Social Change in the Philippines. Londres: Intertext Book.

- 2001. Magosaha : An Ethnography of the Twawi Tawi Sama Dilaut.

Quezon : Ateneo de Manila University Press.

Barrau, Jacques. 1974. "L'Asie du Sud-Est, berceau cultural», Études rurales. Benveniste, Émile. 1966. Problèmes de linguistique générale 1. Paris : Gallimard. —.1969. Problèmes de linguistique générale 2. Paris : Gallimard.

Bloomfield, Leonard. 1933. Language. New York : Holt \& C. 
Boas, Franz. 1911. «Unconscious Characters of Linguistic Phenomena», Handbook of American Indian Languages. Washington : Government Printing Office.

-1916. Tsimshian Mythology, 31st Annual Report of the Bureau of American Ethnology (1909-1910), Washington, DC.

- 1964. "Linguistics and Ethnology», Language in Culture and Society, sous la dir. de Dell Hymes, 15-26. New York : Harper and Row.

Bowlt, John E. 1988. Russian Art of the Avant-Garde Theory and Criticism. Édition revue et corrigée (première édition, 1957). Londres et New York: Thames and Hudson.

Brown, Edward (éd.). 1973. Major Soviet Writers. New York : Oxford University Press.

Chauviré, Christiane. 1995. Peirce et la signification. Introduction à la logique du vague. Paris : Presses Universitaires de France.

Chomsky, Noam. 1957. Syntactic Structures. La Haye : Mouton \& Co.

Conklin, Harold C. 1954. The Relation of Hanunóo Culture to the Plant World, thèse de doctorat. New Haven : Yale University.

—. 1955. «Hanunóo Colors Categories». Southwestern Journal of Anthropo$\log y 11, \mathrm{n}^{\mathrm{o}} 4: 339-344$

—. 1962. "Lexicographical Treatment of Folk Taxonomies». International Journal of American Linguistics 28, $\mathrm{n}^{\mathrm{O}} 2$ : 119-141.

- 1964. «Ethnogenealogical Method», Exploration in Cultural Anthropo$\log y, 25-55$. New-York : McGraw-Hill.

-1980. Folk Classification: A Topically Arranged Bibliography of Contemporary and Background References trough 1971. New Haven : Yale University Press.

-1980. Ethnographic Atlas of Ifugao. New Haven : Yale University Press. - 2007. Fine Description. Ethnographic and Linguistic Essays, sous la dir. de Kuipers et McDermott. New Haven : Yale University Southeast Asia Studies. .

De Saussure, Ferdinand. 1916. Cours de linguistique générale. Paris : Payot.

Dumézil, Georges. 1949. Leçon inaugurale au Collège de France. Paris. .1968. Mythe et épopée, vol. I. Paris : Éditions Gallimard.

-1971. Mythe et épopée, vol. II. Paris : Éditions Gallimard.

Durkheim, Émile et Marcel Mauss. 1903. "De quelques formes primitives de classifications. Contribution à l'étude des représentations collectives», L’Année sociologique, $6: 1-72$.

Fox, Robert B. 1952. «The Pinatubo Negritos. Their Useful Plants and Material Culture». The Philippine Journal of Science 81, $\mathrm{n}^{\circ} 3-4: 173-414$.

Frake, Charles O. 1969. "The Ethnographic Study of Cognitive Systems», Anthropology and Human Behaviour, sous la dir. de Gladwin et Sturtevant, 72-85. Washington : Anthropological Society of Washington.

Goodenough, Ward H. 1957. "Cultural Anthropology and Linguistics», Language and Linguistics, sous la dir. de Garvin, 167-173. Washington : Georgetown University. 
Greenberg, Joseph. 1966. "Language Universals», Current Trends in Linguistics III, sous la dir. de Thomas A. Sebeok, 61-112. La Haye : Mouton.

Gumperz, John J. et Dell Hymes. 1964. The Ethnography of Communication, American Anthropologist, 66, $\mathrm{n}^{\circ}$ 6, deuxième partie (numéro spécial).

Hagège, Claude. 1967. "Documents : Extraits de la correspondance de N. S. Troubetzkoy", La linguistique, 109-136. Paris : Presses Universitaires de France.

. 1985. L'Homme de paroles, contribution linguistique aux sciences humaines. Paris : Fayard.

Harris, Zellig S. 1976. Notes du cours de syntaxe, traduit de l'anglais par Maurice Gross. Paris : Seuil.

Haudricourt, André-Georges. 1972. Problèmes de phonologie diachronique. Paris : SELAF.

—1987. Les pieds sur terre. Paris : Métaillié.

Haudricourt, André-Georges et Claude Hagège. 1978. La phonologie panchronique. Paris : Presses Universitaires de France.

Hockett, Charles F. 1958. Course in Modern Linguistics. New York: Macmillan. Honko, Lauri. 1996. Epics along the Silk Roads. A Unesco Workshop in Turku, Finland, Oral Tradition, $11, \mathrm{n}^{\circ} 1$.

Humboldt, Wilhelm von. 1974 (1836-39). Introduction sur l'œuvre sur le kavi et autres essais. Paris : Seuil.

Husserl, Edmund. 1962. Recherches logiques, Tome second : Recherches pour la phénoménologie et la théorie de la connaissance, traduit de l'allemand par Hubert Elie, 45-81. Paris : Presses Universitaires de France.

Hymes, Dell H. 1962. "The Ethnography of Speaking», Anthropology and Human Behaviour, sous la dir. de Gladwin et Sturtevant, 15-53. Washington : Anthropological Society of Washington.

—. 1964. Language in Culture and Society. A Reader in Linguistics and Anthropology. New York : Harper and Row.

Jakobson, Roman. 1919; 1987. "Futurism», repris dans Roman Jakobson, Language in Literature, ch. II, sous la dir. de Krystyna Pomorka et Stephen Rudy. Cambridge : Harvard University Press, (1 ère éd. : «Futurizm», Isskusstvo, Moskova).

—.1921. «Parts and Wholes in Language», Parts and Wholes, sous la dir. de Lerner. New York : Free Press of Dencoe.

__. 1921; 1973. "Modern Russian Poetry : Vledimir Khlebnikov (Excerpts)", Major Soviet Writers, sous la dir. de Edward J. Brown, (1 ère éd. : Noveyshaya russkaya poeziya. Nabrosok pervy, Prague). New York: Oxford University Press.

- 1953; 1963. «From the Point of View of Linguistics», Supplements to International Journal of American Linguistics, 19:2, Memo 8. - 1956. The Metaphoric and Metonymic Poles. Fundamentals of Language, sous la dir. de Roman Jakobson et Morris Halle. Gravenhage : Mouton. .1963a. «Implications of Language Universals for Linguistics», Universals of Language, sous la dir. de Joseph Greenberg, 263-278. Cambridge : MIT Press. 
1963b. Essais de linguistique générale, traduit et préfacé par Nicolas Ruwet. Paris : Minuit.

.1969. Langage enfantin et aphasie. Paris : Minuit.

.1973. "De la poésie à la linguistique», L'ARC, 18-19.

-1976. Six leçons sur le son et le sens, préface de Claude Lévi-Strauss. Paris : Minuit.

Kroeber, Alfred L. et al. 1953. Anthropology Today, An Encyclopedic Inventory. Chicago : Chicago University Press.

Leroi-Gourhan, André. 1943. L’homme et la matière. Paris : Albin Michel. 1945. Milieu et technique. Paris : Albin Michel.

Lévi-Strauss, Claude. 1949. Les structures élémentaires de la parenté. Paris : Presses Universitaires de France.

. 1950. «Introduction à l'œuvre de Marcel Mauss». Marcel Mauss, Sociologie et anthropologie. Paris : Presses Universitaires de France, IX-LII.

- 1953; 1958. «Linguistique et anthropologie» (Conférence originale en anglais "From the Point of View of Anthropology», Supplements to International Journal of American Linguistics, $19: 2$, Memo 8; en français : Anthropologie structurale, ch. IV, suivi de "Postface aux chapitres III et IV », ch. V, 77-110.

- 1955 "The Structural Study of Myth», "Myth, A Symposium», Journal of American Folklore, 78 : 270, 428-444; en français : "La structure des mythes", Anthropologie structurale, 227-255. Paris : Plon.

_.1958a. Anthropologie structurale. Paris : Plon.

1958b. La geste d'Asdiwal. Paris : Imprimerie nationale.

. 1960a. Leçon inaugurale faite le mardi 5 janvier 1960, Chaire d'anthropologie sociale, Collège de France, Paris.

-1960b. "L'analyse morphologique des contes russes", Cahiers de l'Institut de Science économique appliquée, 9, ISEA, 3-36; International Journal of Slavic Studies and Poetics, 3; repris dans "La structure et la forme", Anthropologie structurale deux, 1973, ch. 8. 139-173.

1962a. La pensée sauvage. Paris : Plon.

-1962b. Le totémisme aujourd'hui. Paris : Presses Universitaires de France. 1964. Mythologiques, Le cru et le cuit. Paris : Plon.

1967. Mythologiques, L'homme nu. Paris : Plon.

-1973. Anthropologie structurale deux. Paris : Plon.

_.1976. «Préface», Six leçons sur le son et le sens de Roman Jakobson, 1-18. Paris : Minuit.

—1983. Le regard éloigné. Paris : Plon.

-1984. Paroles données. Paris : Plon.

-1988. De près de loin. Paris : Odile Jacob.

Lounsbury, Floyd, G. 1969. «Language and Culture», Language and Philosophy, sous la dir. de Hook, 3-29. New York : New York University Press.

Lyons, John. 1981. Language, Meaning and Context. Londres : Fontana Paperbacks.

Macdonald, Charles. 1977. Une société simple. Parenté et résidence chez les $\mathrm{Pa}$ lawan (Philippines). Paris : Institut d'Ethnologie, Musée de l'Homme. 
- 1988. L'éloignement du ciel. Invention et mémoire des mythes chez les Palawan du sud des Philippines. Paris : Maison des Sciences de l'Homme.

Martenot, Alain. 2005. "Une lecture ethnographique de Silungan Baltapa", Le voyage au ciel d'un héros sama, Silungan Baltapa, sous la dir. de Nicole Revel et al. (Éd. trilingue sinama-français-anglais), 149-170. Paris : Geuthner.

Martinet, André. 1955. Économie des changements phonétiques : traité de phonologie diachronique. Berne: Francke.

_.1960. Éléments de linguistique générale. Paris : Collin.

—. 1965. La linguistique synchronique. Paris : Presses Universitaires de France.

Mauss, Marcel. 1950. Sociologie et anthropologie. Paris : Presses Universitaires de France.

Mayakovsky, Vladimir. 1923. For the Voice. Londres : The British Library \& Artists Bookworks.

Merleau-Ponty, Maurice. 1945. Phénoménologie de la perception. Paris : Gallimard.

Nida, Eugene. 1945. "Linguistic and Ethnology in Translation Problems», Word $1, \mathrm{n}^{\circ} 2: 194-208$.

Peirce, Charles S. 1931-58. Collected papers. Cambridge : Harvard University Press.

—.1978. Écrits sur le signe, rassemblés, traduits et commentés par Gérard Deledalle. Paris : Seuil.

Pike, Kenneth. 1954-55. Language in Relation to a Unified Theory of Structure of Human Behaviour. Glendale : Summer Institute of Linguistics.

Polivanov, Evgenij. 1930-31; 1968. «La perception des sons d'une langue étrangère», Travaux du Cercle linguistique de Prague 4, 79-96. Nendeln L: Kraus.

Pouillon, Jean et Pierre Maranda, éds. 1971. Échanges et communications : mélanges offerts à Claude Lévi-Strauss à l'occasion de son soixantième anniversaire, 2 vol. La Haye/Paris : Mouton.

Propp, Vladimir. 1928, 1965. Morphologie du conte, traduit par Marguerite Derrida, Tzvetan Todorov et Claude Khan. Paris : Seuil.

Revel, Nicole. 1983. Kudaman. Une épopée palawan chantée par Usuj (Philippines). Paris : Mouton.

- 1988. Le riz en Asie du Sud-Est: Atlas du vocabulaire de la plante, (Articles, livret du vocabulaire, 78 cartes. Paris : Éditions de l'École des hautes études en sciences sociales.

. 1990-92. Fleurs de paroles. Histoire naturelle Palawan. Paris : PeetersSELAF. (3 vol.) :

I. Les dons de Nägsalad, 1990.

II. La maîtrise d'un savoir et l'art d'une relation, 1991.

III. Chants d'amour / Chants d'oiseaux, 1992.

. 1996. "Kudaman Oral Epic in the Palawan Highland», Oral Tradition 11, $\mathrm{n}^{\mathrm{o}}$ 1, L. Honko ed., Epics Along the Silk Road, «Mental Text and the notion of 'Multiple Draft'», 1st UNESCO Workshop on Epics, Turku, Finland), University of Missouri, 108-132. 
2000. La quête en épouse, The Quest for a Wife. Mämiminbin, (Éd. trilingue palawan-français-anglais et 1 CD). Paris : Éditions Unesco; L'Asiathèque / Langues \& Mondes.

- 2006. "Memory of Voice», Tenth International Conference on Austronesian Linguistics, (10-ICAL), Quezon City. En ligne : <http://www.sil.org/ asia/philippines/ical/papers/revel-Memory\%20of\%2oVoice.pdf>.

_. 2011 [à paraître]. "Le chant de la mémoire», Les lieux de savoir, Tome II, Les mains de l'intellect, sous la dir. de Christian Jacob. Paris : Albin Michel.

Revel, Nicole, Alain Martenot, Harry Arlo Nimmo, et al. 2005. Silungan Baltapa, Le voyage au ciel d'un héros sama / The Voyage to Heaven of a Sama Hero, (Éd. trilingue sinama-français-anglais, 1 DVDrom). Paris : Geuthner,

Ricœur, Paul. 1983. Temps et récit, Tome I, L’intrigue et le récit historique. Paris : Seuil.

-1984. Temps et récit, Tome II, La configuration dans le récit de fiction. Paris : Seuil.

Rousseau, Jean-Jacques. 1776; 1997. Discours sur l'origine et les fondements de l'inégalité parmi les hommes. Paris : Hachette.

_._ 1783; 1990. Discours sur l'origine des langues. Paris : Gallimard. .1771-73; 1983. Le Botaniste sans maître, ou manière d'apprendre seul la botanique, annoté par André-Georges Haudricourt, réédition des «Huit lettres élémentaires sur la botanique» écrites et adressées à Madame Delessert, augmentée de "Fragments pour un dictionnaire des termes d'usage en botanique». Paris : Métaillié.

Sapir, Edward. 1927. The Unconscious : A Symposium, sous la dir. de Dummer, 544-559. New York.

.1951. Selected Writings of Edward Sapir in Language, Culture, and Personality. Berkeley : University of California Press.

1967. Anthropologie 1. Culture et personnalité. 2. Culture, traduit par Christian Baudelot et Pierre Clinquart. Paris : Minuit.

Sebeok, Thomas A. (éd.). 1958. Myth : A Symposium. Bloomington : Indiana University Press.

St-Hilaire, Geoffroy. 1830. Principes de zoologie comparée. Paris : Académie royale des sciences.

Sturtevant, William C. 1964. "Studies in Ethnoscience», Transcultural Studies in Cognition, American Anthropologist, sous la dir. de A. Kimball Romney et Roy d'Andrade 18 : 99-131.

Troubetzkoy, Nicolas S. 1939; 1970. Grundzüge der Phonology, Travaux du Cercle linguistique de Prague. Principes de phonologie, traduit par Jean Cantineau. Paris : Klincksieck.

Tyler, Stephen A., éd. 1969. Cognitive Anthropology. New York : Holt, Rinehart \& Winston.

Veyne, Paul. 1996. Comment on écrit l'histoire. Paris : Seuil.

Whorf, Benjamin L. 1952; 1973. Language, Thought and Reality. Cambridge : MIT.

Zumthor, Paul. 1983. Introduction à la poésie orale. Paris : Seuil. 


\title{
RÉSUMÉ
}

Il nous semble important de montrer comment les affinités intellectuelles qui ont uni Roman Jakobson et Claude Lévi-Strauss ont conduit à des liens théoriques étroits entre la linguistique structurale et l'avènement du structuralisme en anthropologie.

Un essai épistémologique mettant en jeu les principales notions opératoires de ces deux disciplines au cours du XX $\mathrm{X}^{\mathrm{e}}$ siècle en Europe et aux États-Unis permettra de comprendre cette émergence des notions fondamentales et des concepts de la pensée et de l'œuvre lévi-straussienne. Nous proposons de suivre ce développement en quatre temps avant d'accéder aux grands traits de la pensée classificatoire et totémique, à l'analogie comme instrument de perception et de pensée.

Enfin nous témoignerons de deux expériences ethnographiques personnelles en Asie du Sud-est insulaire et de l'influence de nos deux amis dans la pratique d'une recherche en anthropologie linguistique.

\begin{abstract}
It seems important to show how the intellectual affinities that united Roman Jakobson and Claude Lévi-Strauss have led to strong theoretical links between structural linguistics and the advent of structuralism in anthropology.

An epistemological essay involving key operating concepts of these two disciplines in the twentieth century in Europe and the United States will help understand the emergence of the basic notions and concepts in the thought and work of Lévi-Strauss. We propose to follow this development in four stages before arriving at the main features of classificatory and totemic thinking, at analogy as instrument of perception and thought.

Finally we will evoke two personal ethnographic experiences in insular Southeast Asia and the influence of our two friends on the research in linguistic anthropology.
\end{abstract}

